ARTICLE

Received 17 Nov 2015 | Accepted 21 Jun 2016 | Published 5 Aug 2016

DOI: $10.1038 /$ ncomms12321

OPEN

\title{
Stromal Hedgehog signalling is downregulated in colon cancer and its restoration restrains tumour growth
}

Marco Gerling ${ }^{1}$, Nikè V.J.A. Büller ${ }^{2, \star}$, Leonard M. Kirnn ${ }^{1,3, \star}$, Simon Joost ${ }^{1}$, Oliver Frings ${ }^{4}$, Benjamin Englert ${ }^{1,3}$, Åsa Bergström ${ }^{1}$, Raoul V. Kuiper ${ }^{5}$, Leander Blaas ${ }^{1}$, Mattheus C.B. Wielenga ${ }^{2}$, Sven Almer ${ }^{6,7}$, Anja A. Kühl ${ }^{3}$, Erik Fredlund ${ }^{4}$, Gijs R. van den Brink ${ }^{2}$ \& Rune Toftgård ${ }^{1}$

A role for Hedgehog $(\mathrm{Hh})$ signalling in the development of colorectal cancer (CRC) has been proposed. In CRC and other solid tumours, Hh ligands are upregulated; however, a specific $\mathrm{Hh}$ antagonist provided no benefit in a clinical trial. Here we use Hh reporter mice to show that downstream $\mathrm{Hh}$ activity is unexpectedly diminished in a mouse model of colitis-associated colon cancer, and that downstream $\mathrm{Hh}$ signalling is restricted to the stroma. Functionally, stroma-specific $\mathrm{Hh}$ activation in mice markedly reduces the tumour load and blocks progression of advanced neoplasms, partly via the modulation of BMP signalling and restriction of the colonic stem cell signature. By contrast, attenuated $\mathrm{Hh}$ signalling accelerates colonic tumourigenesis. In human CRC, downstream Hh activity is similarly reduced and canonical Hh signalling remains predominantly paracrine. Our results suggest that diminished downstream Hh signalling enhances CRC development, and that stromal Hh activation can act as a colonic tumour suppressor.

\footnotetext{
${ }^{1}$ Center for Innovative Medicine, Department of Biosciences and Nutrition, Karolinska Institutet, NOVUM, Hälsovägen 7, 14183 Huddinge, Sweden.

${ }^{2}$ Tytgat Institute for Liver and Intestinal Research and Department of Gastroenterology and Hepatology, Academic Medical Center, Meibergdreef 69-71, AZ1105 Amsterdam, The Netherlands. ${ }^{3}$ Department of Medicine I for Gastroenterology, Infectious Diseases and Rheumatology, Charité, Campus Benjamin Franklin, Hindenburgdamm 30, 12200 Berlin, Germany. ${ }^{4}$ Department of Oncology-Pathology, Science for Life Laboratory, Karolinska Institutet, 17176 Stockholm, Sweden. ${ }^{5}$ Core Facility for Morphologic Phenotype Analysis, Clinical Research Center, Karolinska Institutet, Hälsovägen 7-9, 14183 Huddinge, Sweden. ${ }^{6}$ Department of Medicine, Solna, Karolinska Institutet, 17176 Stockholm, Sweden. ${ }^{7}$ Center for Digestive Diseases, Karolinska University Hospital, 17176 Stockholm, Sweden. * These authors contributed equally to this work. Correspondence and requests for materials should be addressed to R.T. (email: rune.toftgard@ki.se).
} 
T he Hedgehog (Hh) signalling pathway is one of the major regulators of embryonic development. Later in life, it can drive tumorigenesis: mutations that lead to cellautonomous Hh pathway activation cause nearly all basal cell carcinomas, as well as subsets of medulloblastomas and rhabdomyosarcomas ${ }^{1}$. By contrast, the majority of solid tumours, including colorectal cancer (CRC), rarely carry mutations in $\mathrm{Hh}$ genes $^{2}$. Instead, in CRC, Hh ligand expression is upregulated and conflicting data suggest either a paracrine role for Hh ligands in shaping a tumour-supportive microenvironment ${ }^{3}$ or autocrine pathway activation that promotes metastasis ${ }^{4}$.

Under homeostatic conditions, the main intestinal ligand, Indian hedgehog (Ihh), is secreted by differentiated enterocytes, whereas downstream signalling is activated exclusively in the stroma ${ }^{5}$. In the 'canonical' Hh signalling cascade, binding of the ligand to the inhibitory receptor Patched 1 (Ptch1) leads to derepression of the activating receptor Smoothened (Smo), thereby initiating a signalling cascade that culminates in the stromal activation of the Glioma-associated oncogene (Gli) proteins, Gli1, Gli2 and Gli3 (ref. 5). Gli1 expression is considered the most reliable indicator of downstream pathway activity, whereas $\mathrm{Hh}$ interacting protein (Hhip), Ptchl and its homologue Ptch 2 are further common downstream targets ${ }^{6}$.

The SMO antagonist, vismodegib, has recently been approved for the treatment of basal cell carcinomas ${ }^{7}$. Motivated by the upregulation of $\mathrm{Hh}$ ligands in CRC, a clinical trial with vismodegib added to first-line therapy in metastatic CRC was recently completed, but yielded a negative result ${ }^{8}$. A trial with vismodegib in pancreatic cancer, in which Hh ligands are similarly overexpressed, had an equally discouraging outcome ${ }^{9}$, whereas a further pancreatic cancer study using another SMO antagonist was halted due to an inferior outcome in the inhibitortreated group ${ }^{10}$. Collectively, these clinical data challenge the paradigm of a tumour-promoting stroma shaped by Hh signalling and contest a putative oncogenic role for cancer cell-autonomous Hh activation in these tumour types.

Functionally, the stromal response to the Hh ligand is part of a paracrine loop that controls differentiation of the intestinal epithelium ${ }^{11}$. Diminished Hh signalling evokes an expansion of the intestinal stem cell compartment and leads to impaired enterocyte differentiation, as well as activation of Wnt signalling, the central oncogenic driver pathway in CRC ${ }^{11-14}$.

Given this discrepancy between data suggesting a tumourpromoting role for $\mathrm{Hh}$ in $\mathrm{CRC}$ on one hand and its prodifferentiating role under homeostatic conditions, together with negative results from clinical trials, on the other hand, we sought to get a more precise picture of the role played by $\mathrm{Hh}$ in colorectal tumourigenesis. Using $\mathrm{Hh}$ reporter mice, we provide evidence that downstream $\mathrm{Hh}$ signalling activity is reduced in murine colon tumours. Functionally, diminished Hh signalling promotes colitis-associated colonic tumourigenesis in mice, whereas stroma-specific Hh activation markedly curtails tumour development. Similarly, human CRCs harbour diminished expression of Hh downstream targets despite upregulated expression of the ligand, Sonic Hh $(\mathrm{SHH})$. Together, our data suggest that stromal activation of $\mathrm{Hh}$ signalling can function as a suppressor of colorectal carcinogenesis.

\section{Results}

Reduced stromal Hh signalling in murine colonic tumours. To induce colonic tumours in mice, we employed a chemical model based on injection of the mutagenic agent azoxymethane (AOM), followed by repeated oral treatment with dextran sodium sulphate (DSS) to induce epithelial damage and subsequent colitis (Fig. 1a) ${ }^{15}$. Tumours in this model recapitulate central aspects of human CRCs as they consistently exhibit mutations in the genes for adenomatous polyposis coli $(A p c)$ or catenin $\beta 1(C t n n b 1)^{16}$, and, less frequently, in $\mathrm{Kras}^{17}$. We consider this model particularly well suited to our purposes, as it can be combined readily with Cre-LoxP models to modify epithelial and mesenchymal Hh signalling, and because tumours arise specifically in the colon but not in the small intestine.

To assess downstream Hh signalling activity, we first subjected Gli1 reporter mice harbouring a $\beta$-galactosidase knock-in to Gli1 $\left(\right.$ Gli $^{\text {lacZ } /+}$ mice $\left.{ }^{18}\right)$, to AOM/DSS treatment, and then visualized Gli1 expression with whole-mount X-gal staining. Although non-malignant mucosa stained strongly, X-gal staining was weak to absent in AOM/DSS-induced tumours (Fig. 1b). Histological analysis revealed Gli1 expression exclusively in the stroma and reduced X-gal staining in the tumours (Fig. $1 c, d$ ).

Real-time quantitative PCR confirmed reduced Gli1 expression in tumours from wild-type (wt) mice, with a congruent reduction of the Hh targets, Hhip and Gli2 (Fig. 1e). Remarkably, expression of the receptor, Ptch1, and its homologue, Ptch2, as well as the main intestinal ligand, Ihh, was dissociated from attenuated downstream $\mathrm{Hh}$ signalling (Fig. 1e), whereas all tumours demonstrated increased Wnt activity, as expression of the Wnt targets, Axin2 and Leucine-rich repeat-containing G-protein coupled receptor 5 ( $\operatorname{Lgr} 5)$ was upregulated (Fig. 1e). RNA in situ hybridization (ISH) confirmed that downregulation of stromal Gli1 can occur despite high Ihh expression in the adjacent tumour cells (Fig. 1f).

Given that $\mathrm{Hh}$ downstream signalling in the intestine is exclusively stromal ${ }^{5}$, the reduced expression of downstream $\mathrm{Hh}$ targets could be the result of a reduction in the number of stromal cells. To explore this possibility, we used immunofluorescence (IF) to stain for the intermediate filaments desmin, vimentin and $\alpha$-smooth muscle actin simultaneously. This marker combination covers the majority of normal and cancer-associated noninflammatory stromal cells, for which no specific single marker exists ${ }^{19}$. However, we saw no differences in the amount of stromal cells between normal mucosa and adjacent tumours, indicating that stromal cell loss is not the reason for diminished expression of Hh downstream targets (Supplementary Fig. 1a-c).

Immunohistochemistry (IHC) of $\beta$-catenin in tumours from Gli $^{\text {lacZ/ }+}$ mice revealed its nuclear translocation in areas of low stromal Gli1 expression (Fig. 1g), substantiating a model in which the stromal downstream $\mathrm{Hh}$ signal is diminished in areas of high epithelial Wnt activity. In line with this, we found that the expression of $\mathrm{p}$-Smads1/5-markers of epithelial differentiation driven by bone morphogenetic protein (BMP) signalling that are highly expressed in differentiated enterocytes ${ }^{20}$-correlated spatially with stromal Gli1 (Fig. 1h).

Hence, colonic tumour development in the AOM/DSS model is unexpectedly associated with a diminution in the stromal $\mathrm{Hh}$ response. To explore Hh expression in a different colon cancer model, we probed RNA-sequencing data from tumours in which Apc activity is regulated by a doxycycline-dependent short hairpin RNA (shRNA) (Gene Expression Omnibus (GEO) data set GSE67186 and ref. 21). We found that these non-inflammatory colon tumours recapitulated the expression pattern of the AOM/DSS model, in that expression of the downstream Hh targets, Gli1, Gli2, as well as Gli3, was reduced significantly and was dissociated from expression of the ligands, Ihh (unchanged) and Shh (increased), as well as the receptor Ptch1 (increased) (Supplementary Fig. 2a-c).

Increased tumour burden upon diminished $\mathrm{Hh}$ signalling. In the murine colon, Ihh messenger RNA expression exceeds that of Shh by several orders of magnitude (Supplementary Fig. 2a and refs 22,23), and an Shh conditional knockout model revealed a limited phenotype strictly confined to the terminal ileum ${ }^{24}$. 
To assess whether reduced Hh signalling can promote malignant transformation in the colon, we used a genetic model in which the main intestinal ligand, Ihh, can be knocked out by Tamoxifen (Tam) administration to VillinCreER;Ih flox/flox; $^{\text {f26-LSL-ZsGreen }}$ mice (hereafter $I h h^{\Delta V i l}$ ), simultaneously activating expression of a conditional fluorescent reporter. Although prolonged loss of $\mathrm{Ihh}$ in intestinal epithelial cells leads to intestinal stem cell accumulation, loss of differentiated cells and activation of the Wnt pathway, it does not, by itself, result in adenoma formation ${ }^{12}$. However, when we exposed $I h h^{\Delta V i l}$ mice to

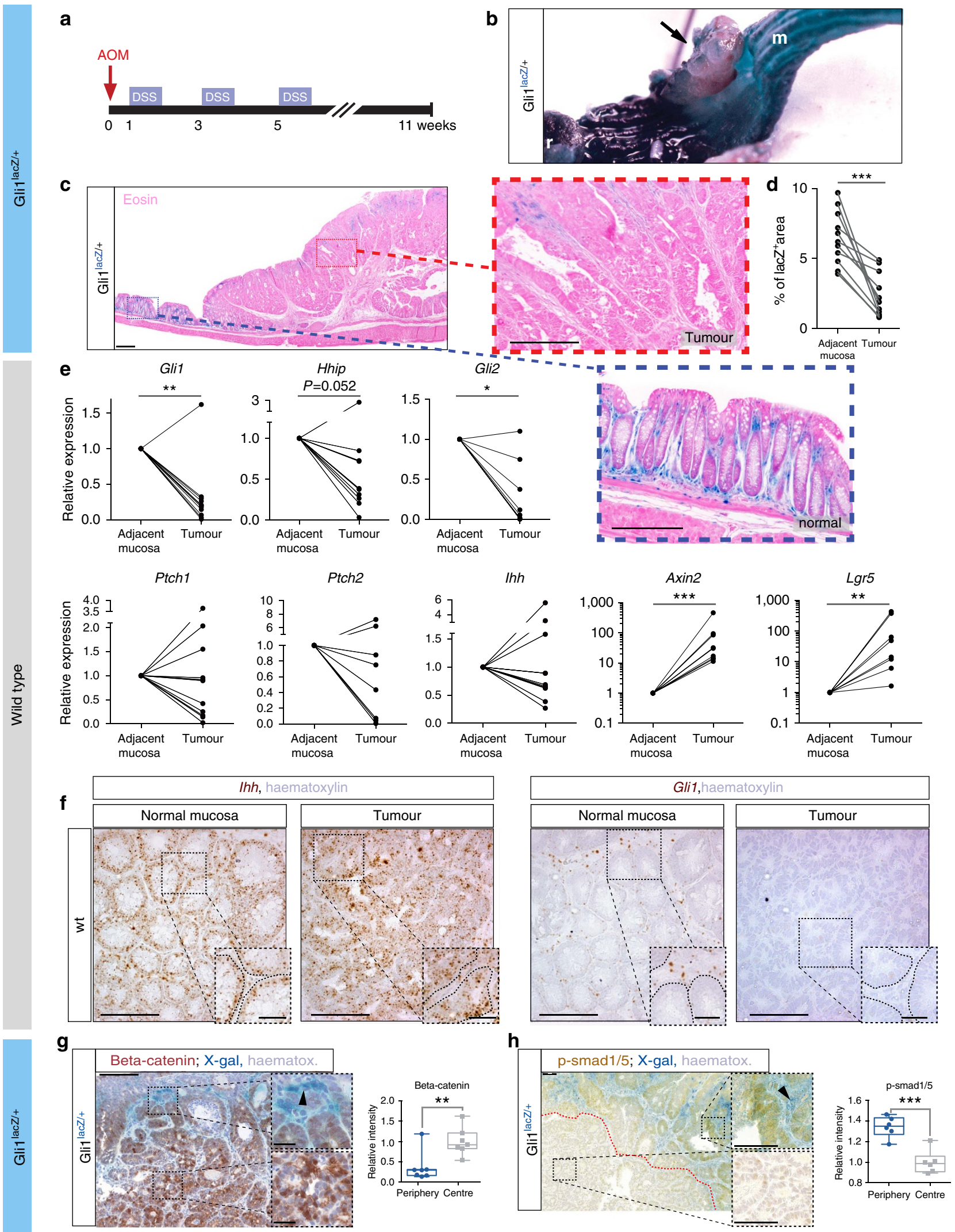


AOM/DSS (Fig. 2a), we found a significant increase in the tumour burden compared with controls (Fig. 2b). The ZsGreen reporter (indicating successful Cre-mediated recombination) persisted throughout normal and tumourous epithelium, demonstrating a permanent loss of Ihh and strongly suggesting that tumour development is not dependent on Ihh expression (Fig. 2c). Indeed, Ihh mRNA was barely detectable in $I h h^{\Delta V i l}$ mice, Shh did not increase significantly and stromal downstream Hh targets were reduced (Fig. 2d and Supplementary Fig. 3a,b). It is worth noting that $I h h^{\Delta V i I}$ mice lost more weight than controls after DSS treatment and had a higher mortality, which may imply that enhanced inflammatory activity contributes, at least partly, to the increased tumour frequency in this model (Supplementary Fig. 3c,d).

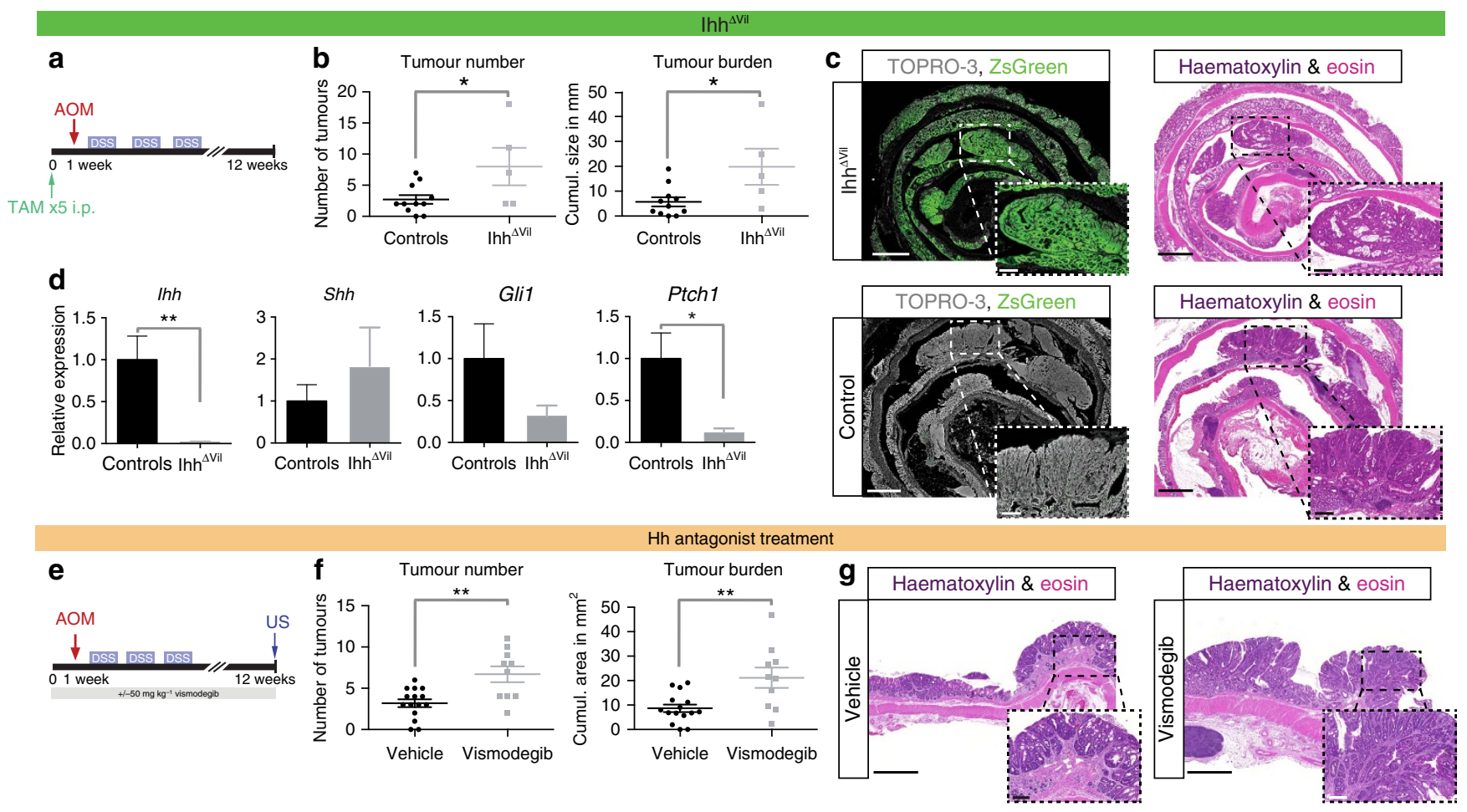

Figure 2 | Reduced Hh signalling increases tumour burden in the AOM/DSS model. (a) Schematic of Tam and AOM/DSS treatment of Ihh ${ }^{\Delta V i l}$ mice. (b) Tumour numbers and tumour burden for $I h h^{\Delta V i l}$ mice $(n=13)$ and controls $(n=11$ littermates that lacked either the VillinCreER allele or floxed Ihh alleles or both): $5 / 13 \mathrm{Ih} h^{\Delta V i l}$ mice and 11/11 controls reached the endpoint; $P=0.0321$ (tumour numbers), $P=0.0215$ (tumour burden; both $t$-tests). (c) Representative confocal image of ZsGreen reporter fluorescence in an $I h^{4 V i l}$ mouse (upper left panel). Persistent reporter expression indicates enduring loss of $\mathrm{th}$ in the tumour. Haematoxylin and eosin (H\&E) staining of consecutive section (upper right panel). Littermate wt controls are shown in the lower panels. Dashed boxes show magnified areas. Nuclear counterstain: TOPRO-3. Scale bars, $1 \mathrm{~mm} / 250 \mu \mathrm{m}$. Representative image of $n=2 \mathrm{Ihh} \mathrm{VV}^{\mathrm{V}}$ mice with $n=8$ tumours, in which all tumours expressed ZsGreen, whereas no zsGreen expression was detected in $n=3$ control mice. (d) Real-time quantitative PCR (RT-qPCR) for the indicated transcripts in Ihh ${ }^{\Delta V i l}$ mice $(n=5)$ and controls $(n=5) ; P=0.003(I h h), P=0.887(S h h), P=0.311(G / i 7)$, $P=0.0142$ (Ptch1; all $t$-tests, $P$-values after multiple test correction $(f d r)$ ). Bars represent mean and whiskers represent s.e.m. (e) Schematic of tumour induction, vismodegib treatment and tumour quantification with US; wt C57BL/6 mice received either $25 \mathrm{mg} \mathrm{kg}^{-1}$ vismodegib or vehicle (DMSO) twice daily, 6 days per week. (f) Tumour numbers and tumour burden for mice treated with vehicle $(n=16)$ or vismodegib $(n=15)$ : 15/16 DMSO-treated and $10 / 15$ vismodegib-treated mice reached the endpoint; $P=0.0014$ (tumour numbers), $P=0.0036$ (tumour burden; both $t$-tests). (g) Representative histology of tumours from the indicated treatment groups (H\&E); scale bars, $1 \mathrm{~mm} / 200 \mu \mathrm{m} .{ }^{\star} P<0.05,{ }^{\star \star} P<0.01$ and ${ }^{\star \star \star} P<0.001$.

Figure 1 | Reduced stromal Hh activity in AOM/DSS-induced colon tumours. (a) Schematic of the AOM/DSS protocol. (b) Representative example of an X-gal-stained tumour in a GlillacZ/ + mouse (from > 20 tumours in 11 mice). Arrow indicates the tumour; r, rectum; m, normal mucosa. (c) Microscopic appearance of an AOM/DSS-induced tumour in a Glil lacZ/ + mouse after X-gal staining (representative of $n=11$ tumours from $n=9$ mice). Magnifications of normal mucosa: blue box and neoplastic tissue: red box; scale bars, 100/50 $\mu \mathrm{m}$. (d) Quantification of X-gal-positive area in tumour and adjacent mucosa $(P=0.0002$; paired $t$-test). (e) Real-time quantitative PCR (RT-qPCR) for the indicated transcripts from tumours and matched normal mucosa, statistics from paired $t$-tests based on the $\Delta C T$ values with multiple test correction (fdr, Benjamini and Hochberg): $P=0.003$ (Gli1), $P=0.052$ (Hhip), $P=0.0232$ (Gli2), $P=0.199$ (Ptch1), $P=0.314$ (Ptch2), $P=0.771$ (Ihh), $P<0.0001$ (Axin2) and $P=0.0016$ (Lgr5); $n=11$ tumours and adjacent mucosa from 11 wt mice; at least 8 matched pairs analysed for each transcript. (f) RNA ISH for Ihh and Gli1 on consecutive sections of the same tumour/mucosa sample; scale bars, $50 / 10 \mu \mathrm{m}$; dashed lines in magnified sections denote epithelial compartment. (g) IHC of $\beta$-catenin in an X-gal-stained GliplacZ/ + tumour indicating mutual exclusivity of epithelial Wnt activation and stromal Gli1; scale bars, 500/50 $\mu \mathrm{m}$. The graph compares the relative staining intensity of the DAB chromophore at the tumour periphery and centre ( $n=7$ tumours, $P=0.006$; box plots; whiskers represent minima and maxima). Arrowhead points to area of positive X-gal staining. (h) IHC of p-Smads1/5 in a tumour from a GlitlacZ/+ mouse. Scale bars, $100 \mu \mathrm{m}$. Quantification of DAB intensity shows an increase at the periphery, in parallel to stromal Gli1 ( $n=6$ tumours, $P=0.0002$; unpaired $t$-tests in $\mathbf{f}$ and $\mathbf{g}$ ); data presented as box-and-whisker plots, whiskers represent minima and maxima. Arrowheads point to cells with positive X-gal staining indicating Gli1 expression. ${ }^{\star} P<0.05,{ }^{\star \star} P<0.01$ and ${ }^{* \star \star} P<0.001$. 
In a subsequent experiment, we used vismodegib to antagonize canonical Hh signalling at the level of the receptor Smo (Fig. 2e). Twice daily administrations of vismodegib (25 mg per $\mathrm{kg}$ body weight) led to a significant reduction in downstream Hh target gene expression (Supplementary Fig. 3e). We treated C57BL/6 wt mice with either vismodegib or vehicle and employed highfrequency ultrasound (US) with intraluminal contrast to assess polypoid colonic lesions at high resolution (Supplementary Movies 1 and 2 and ref. 25). We found that inhibition of canonical Hh signalling with vismodegib had an effect similar to the Ihh knockout, in that it led to more frequent and larger tumours (Fig. 2f,g). However, we observed no significant differences in terms of weight loss or mortality due to colitis (Supplementary Fig. 3f,g).

Taken together, the data demonstrated that canonical downstream Hh signalling is attenuated in murine colorectal carcinogenesis, and that $\mathrm{Hh}$ inhibition can further accelerate tumour development.

Stroma-specific activation of $\mathbf{H h}$ signalling. To visualize $\mathrm{Hh}$ target cells in a near-native setting, we crossed Gli1CreER ${ }^{T 2}$ mice to Rosa26-LSL-tdTomato reporter mice, yielding inducible Gli1CreER ${ }^{T 2}$;Rosa26-LSL-tdTomato mice. Seven days after administration of Tam, we found $t$ thomato ${ }^{+}$cells exclusively residing in the stroma, including a mixed population of cells expressing the cytoskeletal proteins desmin, vimentin and $\alpha$-sma (Fig. 3a).

Gli1 expression remained exclusively stromal after DSS-induced epithelial damage, as indicated by analysis of both the Gli1 lacZ/+ and Gli1CreERT2; tdTomato reporter mice (Supplementary Fig. $4 \mathrm{a}-\mathrm{d}$ ), indicating that downstream $\mathrm{Hh}$ signalling is not activated ectopically in damaged epithelium.

In Gli1CreER ${ }^{T 2}$ mice, Cre is knocked in to the endogenous Gli1 locus ${ }^{18}$; thus, the loss of one functional Gli1 allele alters downstream Hh signalling. To circumvent this problem in further experiments aimed at activating $\mathrm{Hh}$ signalling specifically in stromal cells, we used mice expressing Cre from a transgenic collagen, type I, $\alpha 2$ (Colla2) promoter ${ }^{26}$. Analysis of Tam-induced Colla2CreER;R26-LSL-tdTomato reporter mice confirmed recombination in an exclusively stromal cell population (Fig. 3b) and collagen 1 protein expression overlapped with tdTomato expression in Gli1CreER ${ }^{T 2}$;Rosa26-LSL-tdTomato mice (Fig. 3c). We found that Colla2CreER targets a persistent cell population as indicated by abundant tdTomato ${ }^{+}$fibroblasts 100 days after recombination (Fig. 3d). As the Colla2 transgene is expressed in a wide range of organs ${ }^{26}$, we administered the active metabolite $4 \mathrm{OH}-\mathrm{Tam}$ intraluminally, to minimize systemic effects
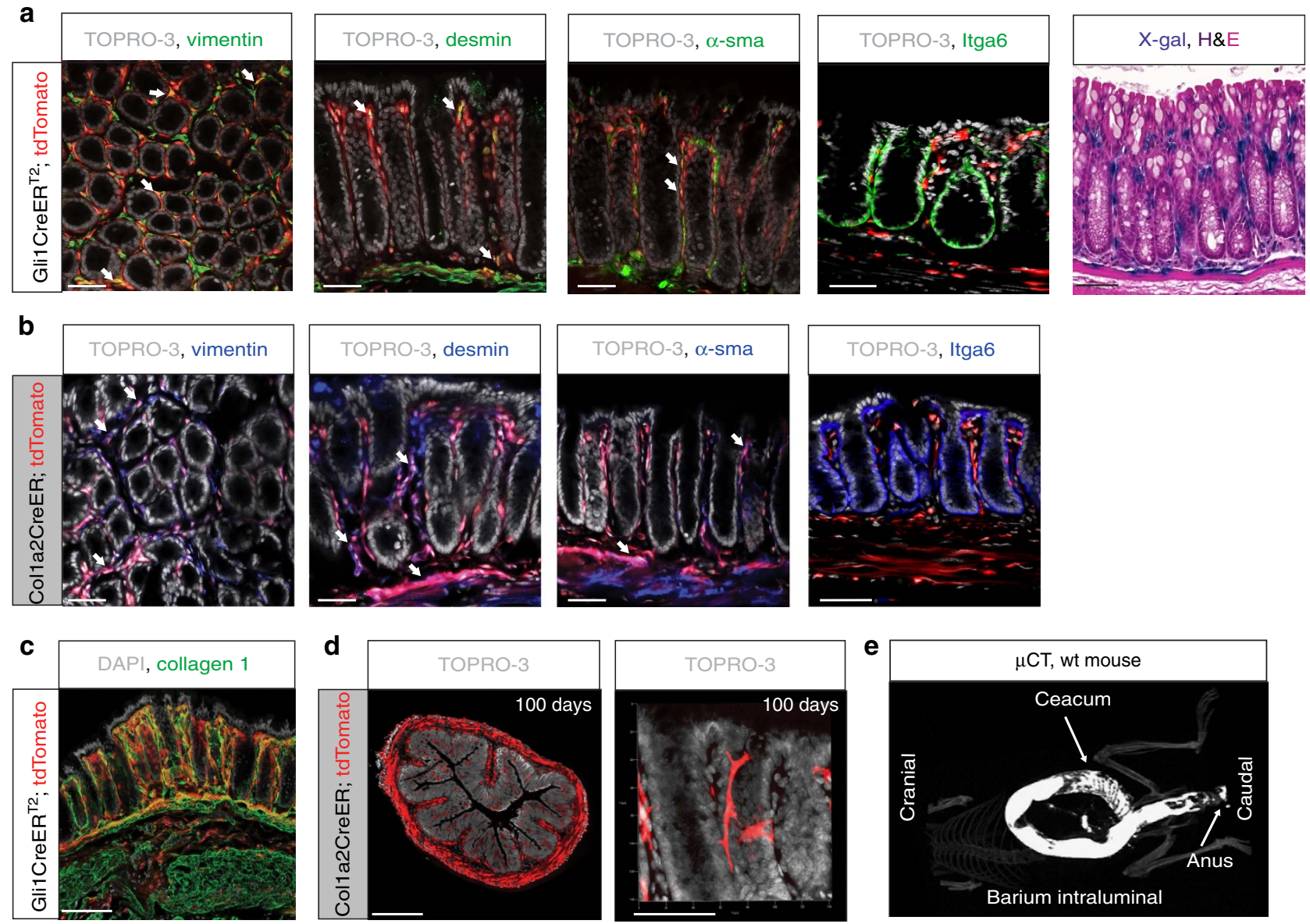

Figure 3 | Stroma-specific Cre-recombination in the murine colon. Initial tracing (7 days after Tam) and IF staining for the stromal proteins vimentin, desmin, $\alpha$-sma and the epithelial protein, integrin $\alpha-6$, with TOPRO-3 as a nuclear counterstain: (a) GliTCreERT2;R26-LSL-tdTomato mouse, representative images from $n=3$ mice. Comparison with the GlitacZ + model in the right panel; scale bars, $50 \mu \mathrm{m}$. (b) Colla2CreER;R26-LSL-tdTomato mouse with representative images from $n>3$ mice for each staining. Scale bars, $50 \mu \mathrm{m}$. (c) 3D rendering of a $25 \mu \mathrm{m}$-thick colon section from a GliTCreERT2;R26-LSLtdTomato mouse 7 days after Tam treatment, stained for collagen-1 (Col1). Overlap of Gli1 and Col1 in yellow. Nuclear counterstain: 4,6-diamidino-2phenylindole (DAPI). Scale bar, $100 \mu \mathrm{m}$. (d) Tomato ${ }^{+}$cells under the control of Colla2CreER 100 days after Tam injection; scale bars, 500/50 $\mu \mathrm{m}$. (e) Barium contrast enema illustrating colonic specificity of intraluminal $4 \mathrm{OH}$-Tam instillations via the rectum (see also Supplementary Fig. 5). 
in subsequent experiments, where we employed Colla2CreERexpressing mice to activate $\mathrm{Hh}$ signalling specifically in the stroma (Fig. 3e and Supplementary Fig. 5).

Hh activation antagonizes colonic tumour development. To activate Hh signalling in Colla2 ${ }^{+}$cells, we crossed Colla2CreER mice to Ptch1neo[A]Ex2[A] mice with LoxP-sites flanking exon 2 of Ptch1 (ref. 27), creating Colla2CreER;Ptch1 $1^{\text {flox } /+}$ mice (hereafter Ptch1 Coll(het) mice). In this model, Tam treatment leads to the knockout of one Ptch1 allele specifically in Colla2expressing stromal cells. Challenged with AOM/DSS (Fig. 4a), Ptch $1^{\text {Coll (het) }}$ mice developed significantly fewer and smaller

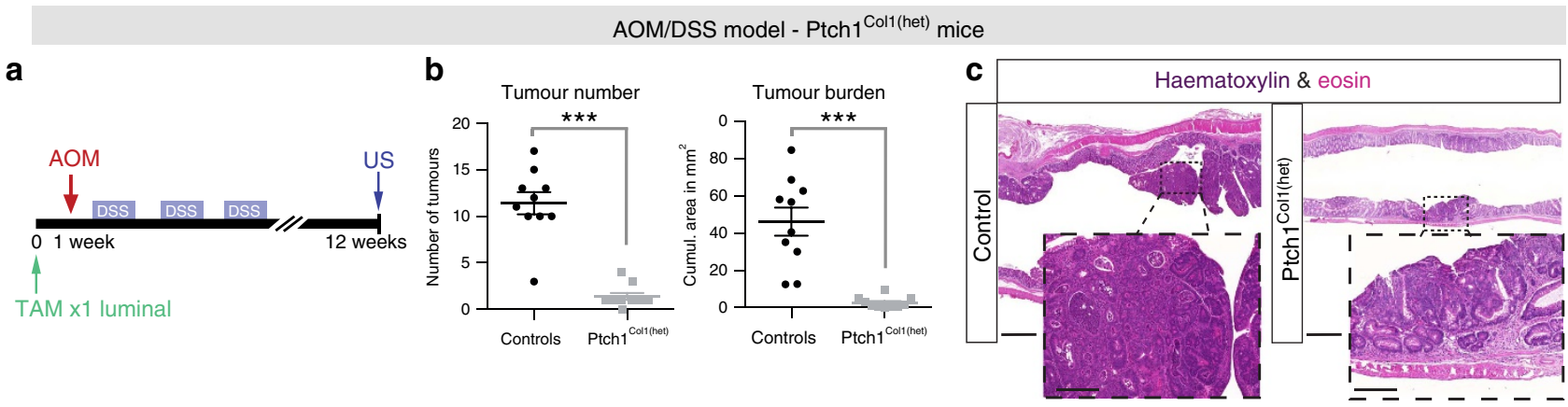

d

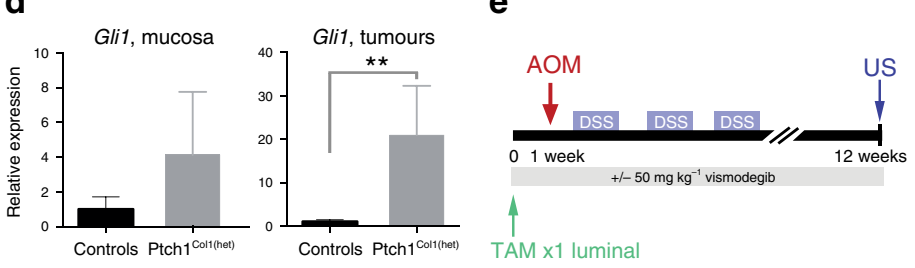

g

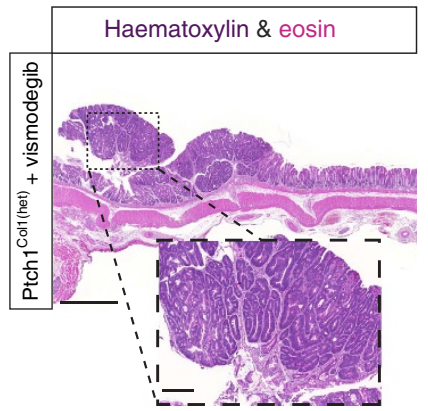

$\mathbf{h}$

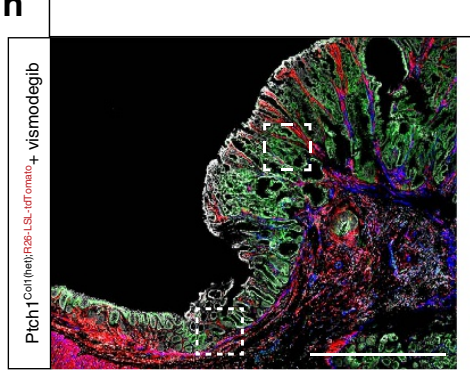

f

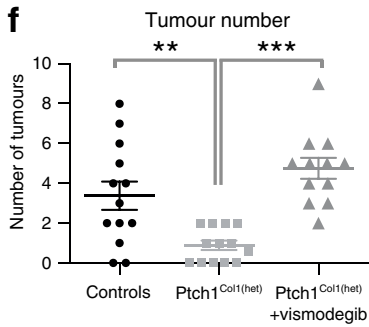

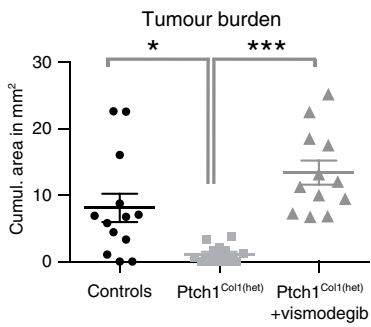

tdTomato, $\alpha$-sma, Ita6, DAPI
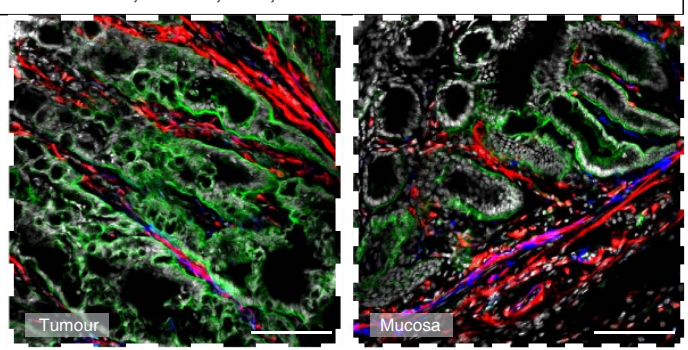

AOM model - Ptch1 ${ }^{\text {Col1 (het) }}$ mice

i
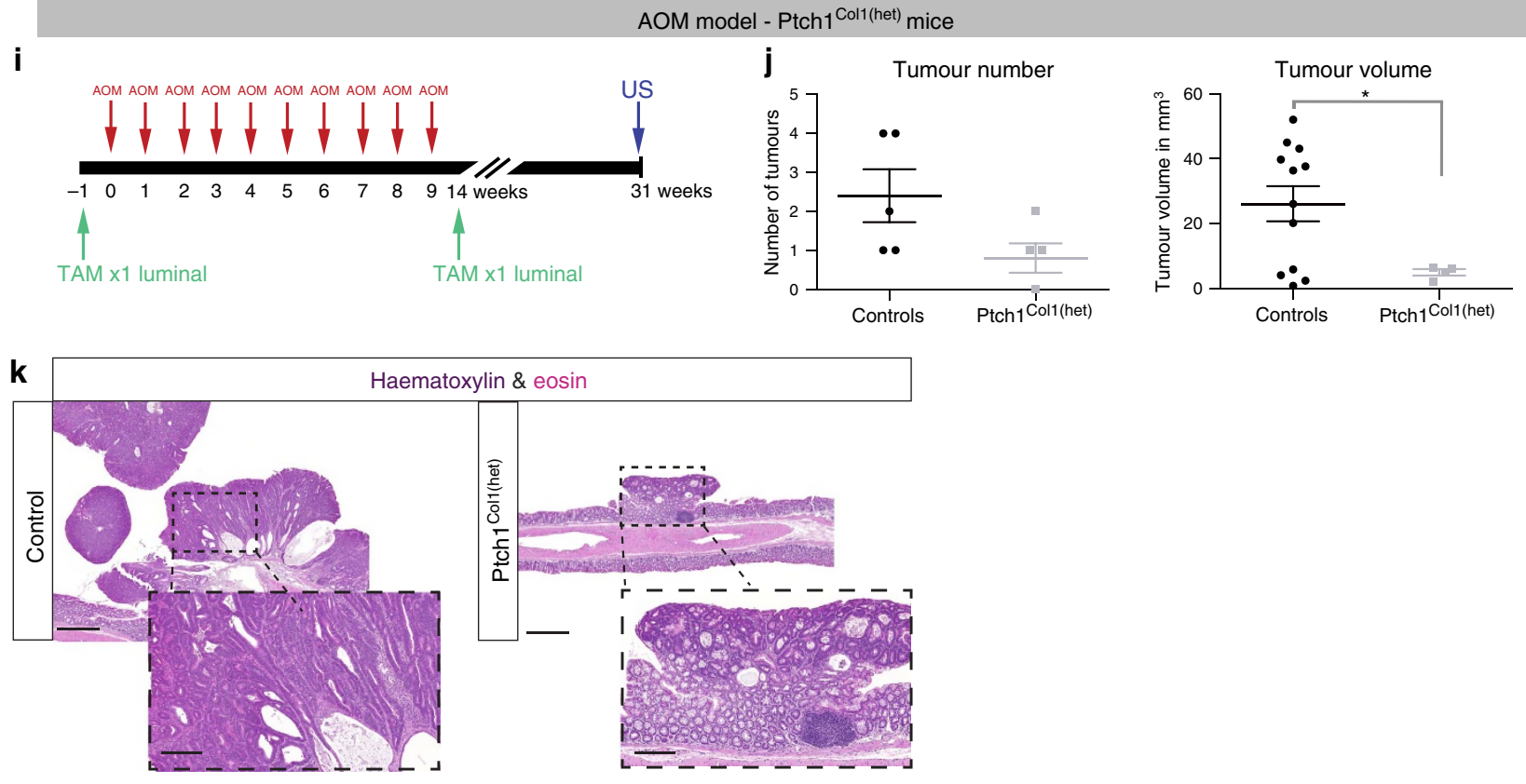
tumours than controls, revealing a protective effect of stromal $\mathrm{Hh}$ activation on colorectal carcinogenesis (Fig. 4b,c). Following the loss of one Ptch1 allele, Gli1 expression increased significantly in tumour tissue, whereas the effect in normal mucosa was less pronounced, probably reflecting background $\mathrm{Hh}$ activity in homeostasis (Fig. 4d). In line with this, we found no phenotypical differences between wt littermates and Ptch $1^{\text {Coll (het) }}$ mice during a 6-month follow-up after recombination (data not shown). Moreover, we observed no differences between Ptch $1^{\text {Coll (het) }}$ mice and control animals with respect to their sensitivity to DSS treatment or mucosal immune cell composition at the endpoint of the AOM/DSS protocol (Supplementary Fig. 6a,b). In tumours from Ptch1 ${ }^{\text {Coll(het) }}$ mice, Gli1 transcripts were enriched in the stromal compartment as expected (Supplementary Fig. $6 c, d$ ), yet the amount of stroma did not differ significantly between tumours from control animals and those from Ptch1 $1^{\text {Coll }}$ (het) mice, as assessed by IF (Supplementary Fig. 6e). Importantly, treatment of Ptchl $1^{\text {Coll (het) }}$ mice with vismodegib (Fig. 4e) overcame the protective effect of $\mathrm{Hh}$ activation, indicating that it is largely mediated in a Smo-dependent manner (Fig. 4f,g). Using Ptch $1^{\text {Coll (het) }}$ mice, treated with vismodegib, and harbouring an additional inducible tdTomato allele, we confirmed that Colla2CreER-targeted cells remained exclusively stromal throughout the AOM/DSS protocol (Fig. 4h), showing that $\mathrm{Hh}$ activation remains confined to the stroma in this model.

The loss of one Ptch1 allele had a marked effect on tumour development, yet it did not detectably influence the course of the DSS-induced colitis. Nevertheless, a role for $\mathrm{Hh}$ in modulating the immune infiltrate in the colon cannot be excluded and previous studies have suggested that stromal Hh signals can act as modifiers of the intestinal immune response ${ }^{11,14}$. To investigate the effect of $\mathrm{Hh}$ activation in a sporadic carcinoma model, we induced tumours in Ptch1 ${ }^{\text {Coll }}$ (het) mice and controls with consecutive AOM injections in the absence of DSS ${ }^{15}$ (Fig. 4i). In accordance with AOM/DSS-treated mice, stromal Hh activation attenuated tumour development in this model (Fig. 4j,k).

Collectively, our results indicated that Hh-driven stromal signals can counteract the neoplastic transformation of overlying epithelial cells.

Diminished BMP inhibitor expression upon Hh activation. To investigate the functional consequences of stromal $\mathrm{Hh}$ activation in greater depth, we performed gene expression analysis on colonic tissue from $P t c h 1^{4 \text { Coll }}$ mice, in which one pulse of Tam induces homozygous inactivation of Ptch1 in the stromal compartment, resulting in activation of Hh signalling in normal mucosa.
Gene expression data confirmed the upregulation of $\mathrm{Hh}$ targets (false discovery rate $(\mathrm{fdr})=0.001)$, dominated by Gli1, Ptch1, Ptch2, and Hhip (Fig. 5a,b upper left panel and Supplementary Tables 1 and 2). Gene set enrichment analysis (GSEA) indicated a significant enrichment of transcripts associated with enterocyte differentiation $(\mathrm{fdr}=0.086)$, such as intestinal alkaline phosphatase (Alpi) or the brush border myosin Ia gene (Myola) (Fig. 5b upper right panel and Supplementary Table 3).

By contrast, Hh activation led to reduced expression of colonic stem cell-associated genes ( $\mathrm{fdr}=0.0313)$, including $\mathrm{Lgr} 5, \mathrm{Cdca} 7$ and $C d k 6$, all of which are restricted to the stem cell compartment at the crypt base (Fig. 5b lower left panel and Supplementary Table 4$)^{28}$. ISH confirmed the reduced expression of Lgr5, whereas Gli1 was increased as expected (Supplementary Fig. 7a,b). These effects of augmented stromal Hh signalling were paralleled by a marked reduction in secreted BMP inhibitors such as Gremlin $1($ Greml) and Noggin $(\mathrm{Nog})(\mathrm{fdr}=0.092)$ (Fig. 5b lower right panel and Supplementary Table 5). Interestingly, Gdf10 (also known as Bmp3b), a member of the BMP family exhibiting Hh-dependent expression, at least in the brain $^{29}$, was among the top 50 upregulated genes (Fig. 5a).

Stroma-specific Hh activation entailed further changes, including alterations in known modifiers of intestinal tumourigenesis. For example, the mesenchymal factor, Foxl1, which was upregulated in $P$ tch $1^{\Delta \text { Coll }}$ mice (Fig. 5a), is directly controlled by Gli proteins $s^{30}$ and attenuates tumour development in a small intestinal adenoma model ${ }^{31}$. As a further example, the gene encoding plasma glutathione peroxidase (Gpx3), which has a protective effect in the AOM/DSS model ${ }^{32}$, was similarly upregulated (Fig. 5a).

To identify genes that show robust alterations in expression as a result of Hh activation specifically in stromal cells, we analysed two independent data sets: (i) human colonic fibroblasts (Fig. 5c,d) and (ii) murine intestinal mesenchymal cells (Fig. 5e,f), treated with Hh ligands in vitro (based on the GEO data sets GSE17840 and GSE29316 (refs 14,33)). In both cases, activation of canonical Hh signalling was paralleled by significant upregulation of Foxl1/FOXL1 and significant downregulation of the BMP inhibitor Grem1/GREM1 (Fig. 5c-f). Other BMP inhibitors such as Nog/NOG, Chrdl1/CHRDL1 and Sostdc1/SOSTDC1 were significantly downregulated in one of the data sets, but not in both (Supplementary Table 6). Based on these data, we revisited tumours from Ptch1 ${ }^{\text {Coll (het) }}$ mice, in which Gli1 expression is increased (Fig. 4d), and analysed the expression of selected BMP inhibitors using real-time quantitative PCR. In line with the in vitro studies, we found that Grem 1

Figure 4 | Stromal Hh activation attenuates colonic tumour development. (a) Experiment schematic; controls comprise littermates lacking either the floxed Ptch1 allele or the Cre recombinase, or both. (b) Tumour numbers and cumulative tumour sizes in controls ( $n=12$ of which 10 reached the endpoint) and Ptch1 ${ }^{\text {Col1 (het) }}$ mice ( $n=12$ of which 10 reached the endpoint). $P<0.0001$ (for both tumour number and burden, $t$-tests). (c) Histology (haematoxylin and eosin (H\&E)) of representative tumours from each group presented in b); scale bars, $1 \mathrm{~mm} / 200 \mu \mathrm{m}$. (d) Gli1 mRNA expression in normal mucosa ( $n=8$ controls and $n=6$ Ptch $^{\text {Coll (het) }}$ mice; $P=0.235$ ) and tumours $\left(n=10\right.$ tumours from controls and $n=9$ tumours from $P$ tch ${ }^{\text {Coll }}$ (het) mice; $\left.P=0.002\right)$; t-test statistics on $\Delta C T$ values. Bars represent means and whiskers represent s.e.m. (e) Schematic of vismodegib administration ( $25 \mathrm{mg} \mathrm{kg}{ }^{-1}$ body weight twice daily, 6 days per week), Tam administration and tumour induction. (f) Tumour numbers and cumulative sizes in controls (littermates as defined in $\mathbf{b}$ that received Tam and twice daily injections of dimethyl sulfoxide (DMSO); $n=17$ of which 13 reached the endpoint), Ptch ${ }^{\text {Coll (het) }}$ mice $(n=13$ of which all reached the endpoint) and $P$ tch ${ }^{\text {Coll (het) }}$ mice (treated with vismodegib twice daily, 6 days per week, $n=19$ of which 12 reached the endpoint). $P$-values from one-way analysis of variance with Tukey's multiple comparison test are indicated. (g) Representative histology (H\&E staining) of a Ptch7 Coll(het) mouse treated with vismodegib. (h) Confocal image of a Ptch ${ }^{\text {Col1(het) }}$ mouse with an inducible R26-LSL-tdTomato reporter, treated with vismodegib. TdTomato ${ }^{+}$ cells are exclusively stromal in tumour (middle panel representing magnification of boxed area in the left panel) and normal tissue (right panel magnification); stromal marker: $\alpha$-smooth muscle actin ( $\alpha$-sma) (blue); epithelial marker: It $\alpha 6$ (green); nuclear stain: 4,6-diamidino-2-phenylindole (DAPI). Scale bars, $1 \mathrm{~mm} / 100 \mu \mathrm{m}$. Representative of $n=4$ tumours in $n=2$ mice. (i) Schematic of AOM injections and Tam administration (repeated as Col1a2 ${ }^{+}$ progeny were known to persist at least for up to 100 days; Fig. 3d). (j) Tumour numbers and volumes for Ptch ${ }^{\text {Colinhet) }}$ mice and controls ( $n=6$ littermates as defined in $\mathbf{b}$, of which five (controls) and four (AOM) reached the endpoint; $P=0.143$ and $P=0.045$, respectively, $t$-tests). (k) Representative histology of the AOM-induced tumours for the indicated genotypes. Scale bars, $1 \mathrm{~mm} / 200 \mu \mathrm{m}$. Whiskers represent s.e.m.; ${ }^{\star} P<0.05$, ${ }^{\star \star} P<0.01$ and ${ }^{\star \star \star} P<0.001$. 


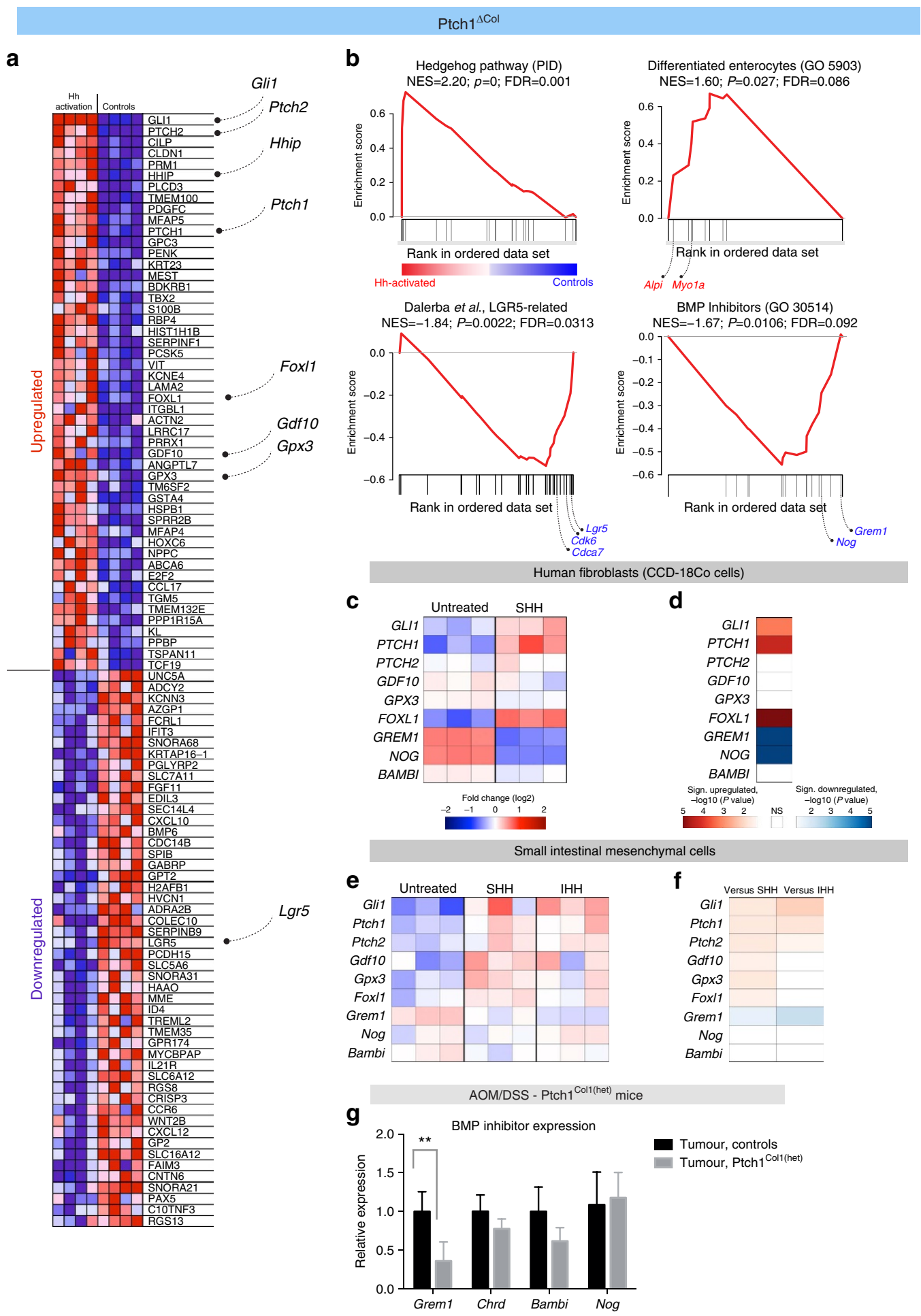

Figure 5 | Transcriptional changes upon Hh activation. (a) Heatmap of the 50 highest ranked genes (using the GSEA algorithm ${ }^{58}$ ) in the murine colon upon knockout of stromal Ptch1 in Ptch ${ }^{4 \text { Coll }}$ mice $(n=4)$ versus controls lacking Cre recombinase $(n=4) 7$ days after a single dose of 5 mg Tam i.p. (b) GSEA indicates activation of canonical Hh signalling (left upper panel); enrichment of genes associated with enterocyte differentiation (right upper panel, gene ontology (GO) term, GO:0005903) and downregulation of colonic stem cell-associated genes (left lower panel) in parallel with loss of secreted BMP inhibitors (right lower panel). Normalized enrichment score (NES), P-value and fdr are indicated in each panel; gene lists are presented in the Supplementary Material. (c) Gene expression of indicated transcripts in the human colon fibroblast cell line, $\mathrm{CCd}-18 \mathrm{Co}$, treated with $1 \mu \mathrm{g} \mathrm{ml}{ }^{-1} \mathrm{SHH}$ or vehicle for $72 \mathrm{~h}$ (data derived from GEO29316 (ref. 33)). (d) Illustration of significant changes in gene expression (fdr adjusted); note highly significant downregulation of GREM1 and NOG, and upregulation of FOXL1. (e) Gene expression in cultured intestinal mesenchyme from E18.5 mouse embryos treated with Ihh or Shh amino-terminal polypeptide $\left(2.5 \mathrm{\mu g} \mathrm{ml}^{-1}\right)$ or vehicle for $24 \mathrm{~h}$ (data derived from GEO17840); scale as in c. (f) Illustration of significant changes in gene expression (fdr adjusted); Grem1 was downregulated by both Shh and Ihh treatment; upregulation of Gdf10, Gpx3 and Fox/1 was significant in Shh-treated mesenchyme. Scale as in d. (g) mRNA expression of the indicated transcripts in tumours of Ptch ${ }^{C o l 1(h e t)}$ mice $(n>5)$ and tumours from control mice (lacking Cre recombinase, floxed Ptch1 alleles or both; $n>8$ ); $P$-values from $t$-tests with fdr correction indicated, $P=0.001$ (Grem1), $P=0.558$ (Nog), $P=0.603$ (Chrd), $P=0.206$ (Bambi) and ${ }^{\star \star} P<0.01$. 

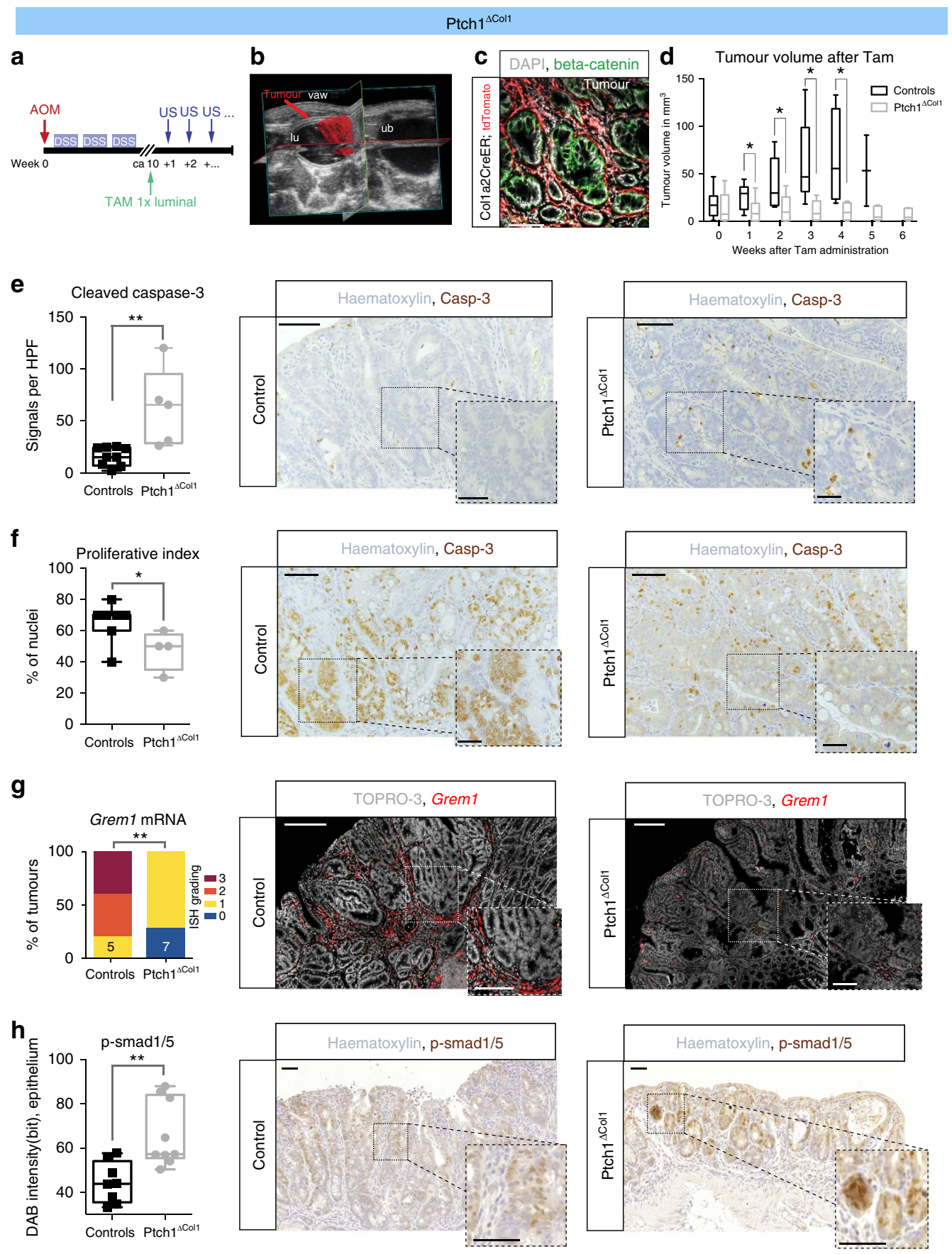

Figure 6 | Growth arrest of established murine tumours upon stromal Hh activation. (a) Protocol schematic. (b) Example of a 3D $\mu$ US reconstruction; tumour volume (arrow) is highlighted in red, other hypoechoic (black) structure is the urinary bladder (ub); lu: colonic lumen, filled with US gel; vaw: ventral abdominal wall. (c) Tomato ${ }^{+}$cells in an AOM/DSS-induced tumour from a Col7a2CreER;R26-LSL-tdTomato mouse 3 days after Tam administration; $\beta$-catenin (green) marks tumour cells; nuclear stain: 4,6-diamidino-2-phenylindole (DAPI); scale bar, $100 \mu$ m. (d) Tumour size as measured by $\mu$ US after Tam instillation (week 0). A total of $n=5$ Ptch $^{4 \text { Coll }}$ mice with $n=11$ tumours and $n=7$ controls (littermates lacking Cre recombinase, floxed Ptch1 alleles or both) with $n=12$ tumours are included; 1 to 5 tumours per mouse were analysed. The adjusted $P$-values (fdr) from $t$-tests are indicated: $P=0.406$ (week 0), $P=0.022$ (week 1), $P=0.029$ (week 2), $P=0.026$ (week 3), $P=0.026$ (week 4) and $P=0.078$ (week 5). Box-and-whisker plots: whiskers represent minima and maxima. In the control group, mice had to be killed because of bleeding per anus, leaving 2 tumours for US analysis in week 5 and none in week 6. (e) Quantification of cleaved caspase-3 positivity per high-power field (original magnification $\times 400$ ): controls ( $n=9$ tumours),

$P t c h{ }^{4 \mathrm{Col}}$ mice ( $n=5$ tumours), $P=0.0034$ ( $t$-test). Middle and right panels show representative IHC images for similar-sized tumours 3 weeks after Tam treatment for the indicated genotypes. Scale bars, $20 \mu \mathrm{m}$. (f) Proliferative index as a percentage of Ki67 ${ }^{+}$nuclei for $n=7$ controls and $n=4$ tumours from $P t c h 7^{4 \mathrm{Col} 1}$ mice, $P=0.0467$ ( $t$-test) and representative images (middle and right panels). Scale bars, 50/20 $\mu \mathrm{m}$. (g) ISH semi-quantification of Grem 1 in control mice ( $n=5$ tumours) and Ptch ${ }^{4 C o l l}$ mice ( $n=7$ tumours). Stacked bars represent the frequency of intensity grading; $P=0.0076$ (Mann-Whitney test), middle and right panels show representative images for the two groups; scale bars, $200 / 100 \mu \mathrm{m}$. (h) Quantification of p-Smad1/5 IHC in control mice ( $n=8$ tumours) and $P t c h 7^{4 C o l 1}$ mice $(n=9$ tumours); $P=0.0028$ ( $t$-test), middle and right panels show representative images for the two groups; scale bars, $50 \mu \mathrm{m}$; (e-h) boxed areas show magnified regions. ${ }^{\star} P<0.05$, ${ }^{\star \star} P<0.01$ and ${ }^{\star \star \star} P<0.001$. 
expression is downregulated in tumours from Ptch $1^{\text {Coll(het) }}$ mice compared with controls (Fig. 5g).

Together, the data indicated that stromal $\mathrm{Hh}$ activation leads to complex transcriptional changes with a prominent, but not exclusive, role for the modulation of BMP signalling. These changes result in restriction of the colonic stem cell state signature and induction of epithelial differentiation markers.

Hh activation blocks the growth of established tumours. AOM/DSS treatment of Ptch $1^{\Delta \text { Coll }}$ mice led to severe morbidity and mortality, precluding their use for the study of tumour initiation (Supplementary Fig. 7c,d,e). Without AOM/DSS treatment, Ptch $1^{\Delta \text { Coll }}$ mice had to be killed several weeks after Tam treatment, as they developed large, cystic stromal tumours predominantly at the ileoceacal junction, which occasionally ruptured or led to gross abdominal distension (Supplementary Fig. 7f,g). Similar tumours have been described in a related model, in which Ptch1 was specifically inactivated in a more restricted stromal compartment ${ }^{34}$.

Nevertheless, we were able to induce tumours with AOM/DSS in $P$ tch $1^{\Delta C o l 1}$ mice that had not received Tam previously and we used this model to activate Hh signalling in established tumours. It has recently been shown that high levels of Wnt signalling are necessary for tumour maintenance, and that restoration to homeostatic Wnt levels causes tumour regression ${ }^{21}$. This prompted us to ask whether activated stromal $\mathrm{Hh}$ signalling might restrain tumour growth in a similar manner. Once AOM/DSS-induced lesions of similar sizes were detected, we used three-dimensional (3D) $\mu \mathrm{US}$ to monitor tumour volumes over time in Ptch $1^{\Delta \text { Coll }}$ mice and control mice after Tam administration (Fig. 6a,b). Using Col1a2CreER;R26-LSL-tdTomato mice, we confirmed that tdTomato ${ }^{+}$cells were directly adjacent to the tumour cells (Fig. 6c).

One week after Tam treatment, delayed growth was evident in tumours from Ptch $1^{\Delta \text { Coll }}$ mice compared with littermate controls (Fig. 6d). Over the course of several weeks, tumours with Hh-activated stroma frequently entered permanent macroscopic growth arrest or, in the case of smaller lesions, showed regression, whereas tumours in control animals progressed (Fig. 6d and Supplementary Movies 1 and 2). IHC analysis of cleaved caspase-3 (Fig. 6e) and Ki67 (Fig. 6f) indicated increased tumour cell apoptosis and diminished proliferative activity, respectively, following stromal $\mathrm{Hh}$ activation.

To assess whether Grem1 is reduced as a consequence of stromal $\mathrm{Hh}$ activation in the tumours, we used RNA ISH and semi-quantitatively evaluated the staining intensity ${ }^{35}$. Although Grem1 was strongly expressed in the tumour stroma of control mice, expression was significantly reduced in $P t c h 1^{\Delta C o l 1}$ mice (Fig. 6g). In addition, tumour-epithelial p-Smad1/5 staining was consistently more abundant in tumours from Ptch $1^{\Delta \text { Coll }}$ mice, indicating increased epithelial BMP downstream activity as a result of the augmented stromal Hh signal (Fig. 6h).

Diminished downstream $\mathrm{Hh}$ response in human colon cancer. Based on the unexpected expression patterns of Hh pathway members in murine tumours, we decided to re-assess $\mathrm{Hh}$ expression in human CRC in more detail, including the pathways' downstream targets. To this end, we first analysed microarray gene expression data from The Cancer Genome Atlas (TCGA), representing 155 colon cancer patients ${ }^{2}$. Although $\mathrm{SHH}$ was upregulated in carcinomas as previously reported ${ }^{3}, I H H$ was moderately downregulated (Fig. 7a). As in the mouse model, the downstream targets, GLI1 and HHIP, were downregulated (Fig. 7b) and, strikingly, no correlation between GLII and $\mathrm{Hh}$ ligand expression was observed (Fig. 7c). In addition, we found that the BMP targets, ID1 and ID2, were significantly downregulated in the carcinomas, whereas GREM1 and NOG expression increased, albeit not significantly in the case of GREM1 (Fig. 7d). Moreover, expression of the BMP ligands, GDF10/BMP3b and BMP5, was reduced significantly (Fig. 7d).

In some colon cancer cell lines with mutations in KRAS or BRAF, cancer cell-autonomous GLI1 activation has been described $^{36}$. However, we found no correlation between the presence of mutated KRAS or BRAF and GLI1 mRNA levels (Supplementary Fig. 8a). Instead, there was a weak but significant correlation between mutations in the APC gene and low GLI1 levels (Supplementary Fig. 8a).

GLI1 mRNA levels correlated well with stromal gene expression signatures in the TCGA data set (Supplementary Fig. 8b), indicating that activation of downstream Hh signalling is largely confined to the stromal compartment. To confirm this further, we probed gene expression data from CRC patient-derived xenografts, in which murine stromal cells replace the human stroma after transplantation (based on GEO GSE56710 and GSE35144 data sets) ${ }^{37}$. In the xenografts, expression of the ligands, $S H H$ and $I H H$, was exclusively epithelial, whereas downstream signalling was largely restricted to the stroma, as transcripts of GLI1 (>75\%) and GLI2 (95\%), as well as HHIP (100\%) were predominantly or exclusively murine (Fig. 7e).

To assess gene expression in the different cellular compartments in a more quantitative manner, we analysed Hh and BMP pathway components in CRCs sorted for epithelial cells $\left(\mathrm{CD} 45^{-}\right.$, Epcam $\left.{ }^{+}\right)$, leukocytes $\left(\mathrm{CD}^{+} 5^{+}\right.$, Epcam $\left.^{-}\right)$and stromal cells $\left(\mathrm{CD}^{-} 5^{-}, \text {Epcam }^{-} \text {; based on the GEO39395 data set }\right)^{38}$. We found a similar pattern to that in the xenografts, such that downstream Hh targets were enriched in the stroma to a highly significant degree, whereas ligand expression was epithelial (Fig. 7f,g). GREM1 and the BMP ligand BMP5 were enriched significantly in the stromal fraction, in keeping with the results from the mouse model, whereas tumour cells expressed BMP4 in several cases. Of note, the $\mathrm{Hh}$ co-receptor CDON and PTCH1 were expressed in the tumour cells at a level similar to that seen in the stroma, although this did not lead to increased expression of Hh downstream targets in the epithelial compartment (Fig. 7f).

In summary, the results obtained from different CRC cohorts demonstrated that human colon cancer exhibits a marked decrease in downstream $\mathrm{Hh}$ target gene expression, which is predominantly, if not exclusively, of a stromal nature.

\section{Discussion}

Under homeostatic conditions, the mode of intestinal Hh signalling is exclusively paracrine, from the ligand-secreting epithelium to the receptive underlying stroma ${ }^{11,39}$. However, a role for cell-autonomous downstream $\mathrm{Hh}$ activity in colon cancer cells has been suggested, in particular at later tumour stages, in which $\mathrm{Hh}$ activation drives metastasis ${ }^{4,40}$.

In the current study, our analyses of stromal and epithelial gene expression strongly suggest that there is little, if any, cell-autonomous $\mathrm{Hh}$ downstream activity in colon cancer cells (Fig. 7e,f). Together with the marked overall downregulation of GLI1 and HHIP (Fig. 7b), these results provide consistent evidence that stromal downstream Hh activity is diminished in colon cancer. However, as some CRC cell lines are dependent on GLI1 (refs 36,40), it is possible that downstream Hh signalling might play a role in a tumour cell subpopulation. On the other hand, most CRC cell lines do not express a complete set of Hh downstream targets ${ }^{41}$. Aside from a role for canonical $\mathrm{Hh}$ signalling, it has previously been suggested that Hh ligands can prevent colon cancer cells from entering apoptosis by binding to the dependence receptor, $\mathrm{CDON}$, in an autocrine manner (independent of the role of $\mathrm{CDON}$ as a $\mathrm{Hh}$ co-receptor) ${ }^{42}$. 
As we find that $C D O N$ is expressed in the tumour cell compartment (Fig. 7f), this leaves a possible tumour-promoting role for a non-canonical SHH/CDON/PTCH1 axis, acting independently of downstream Hh signalling (Fig. 7h).

In contrast to carcinomas, human colonic adenomas from patients with familial adenomatous polyposis as well as small intestinal adenomas from $A p c^{l o x 15}$ mice exhibit increased levels of IHH/Ihh and GLI1/Gli1 (ref. 12). In Apc $c^{\text {lox15 }}$ mice the presence of $I h h$ is required for adenoma development ${ }^{12}$, whereas in the AOM/DSS model Ihh loss fuels tumourigenesis (Fig. 2a). Importantly, treatment of later stage small intestinal murine adenomas with Hh inhibitors promoted, rather than restricted,

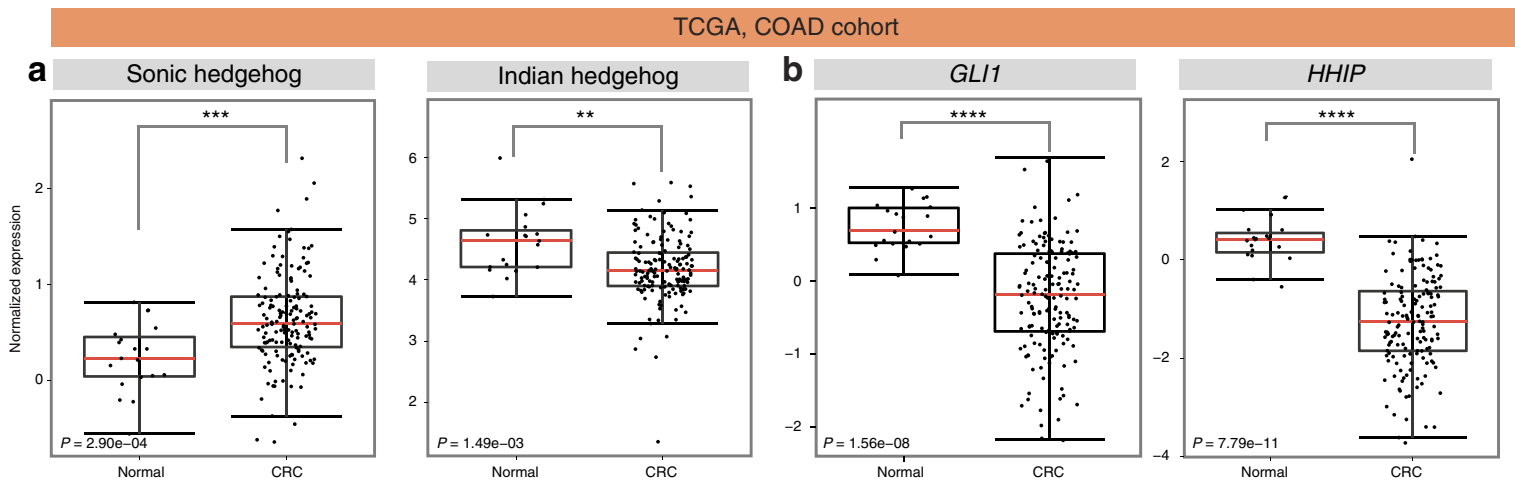

C Correlation of GL/1 expression to Hh components

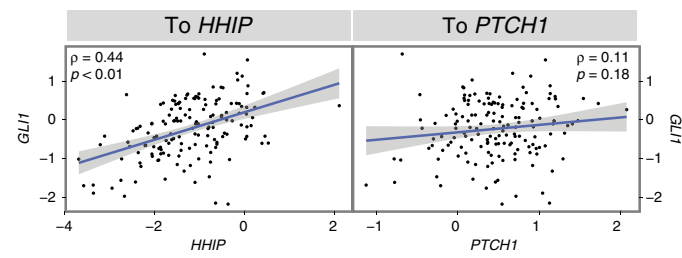

d

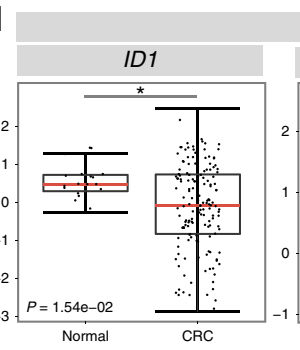

BMP signalling
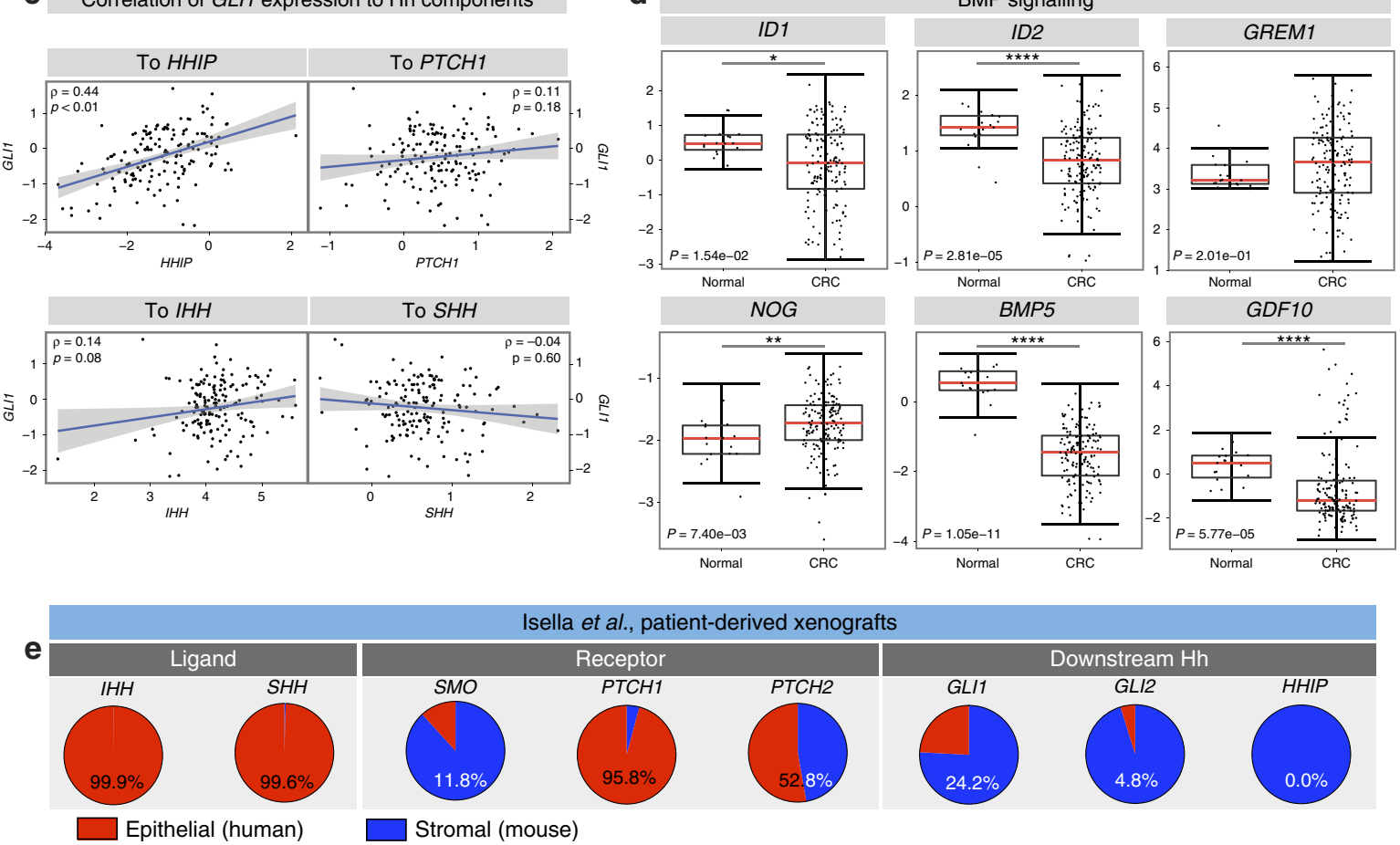

Calon et al., sorted cells from CRC tissue
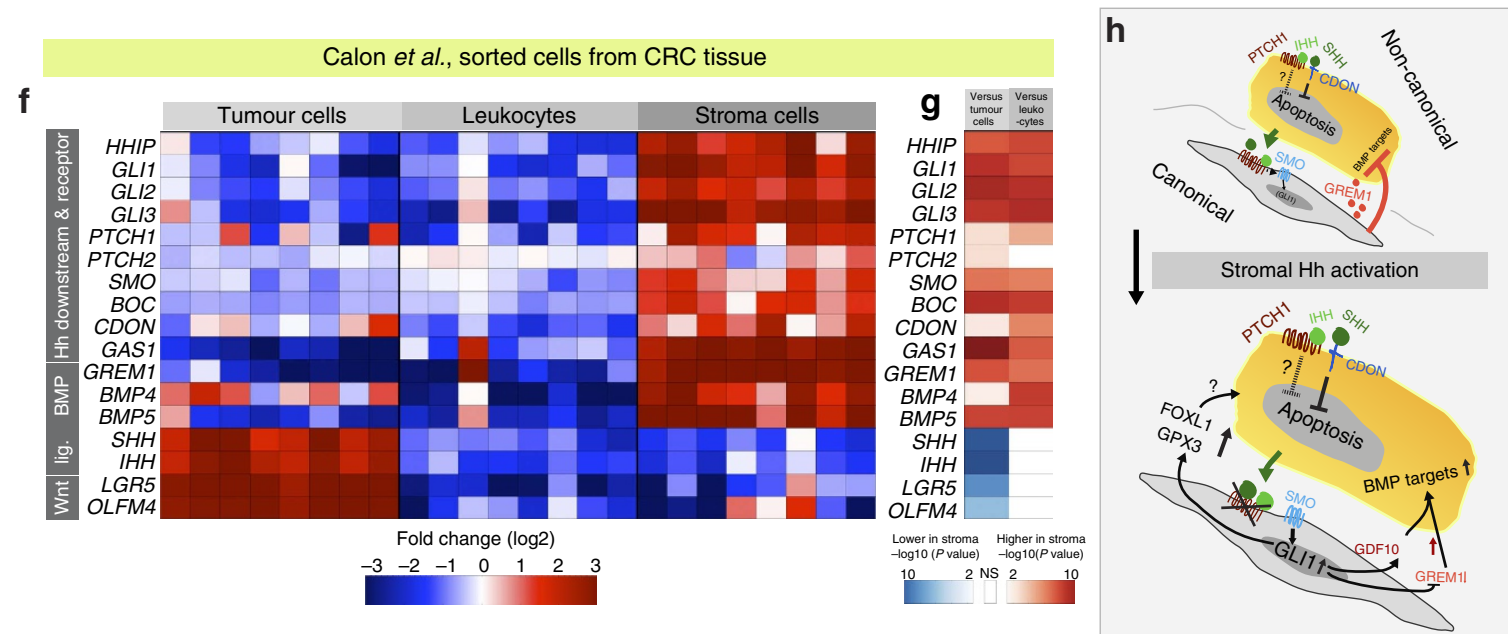
tumour growth in the $A p c^{\text {min }}$ model $^{12}$. Almost all functional data on intestinal Hh signalling from in vivo models have thus far been acquired in the small intestine $\mathrm{in}^{11-14,43}$. However, the tissue architecture and cellular composition of the small intestine differ considerably from those of the colon, in which the vast majority of human intestinal cancers arise. It is therefore noteworthy that colonic tumours in mice, whether they are induced chemically with AOM/DSS (Fig. 1), or genetically, as in the ShApc model ${ }^{21}$ (Supplementary Fig. 2), recapitulate the Hh expression pattern seen in human CRCs ${ }^{12}$. Hence, tissue-specific properties of the small intestine and colon may explain the different requirements in the two locations with regard to dependence on Hh signalling.

Across various colon cancer mouse models and in human CRC, downstream $\mathrm{Hh}$ activity can be lost despite increased ligand expression (Figs 1 and 7, and Supplementary Fig. 2). This might be the result of alterations in stromal gene expression programmes that render stromal cells indifferent to the epithelial ligand $^{44}$. An alternative explanation has recently been proposed by Shyer et al. ${ }^{45}$, who found that during development Hh ligands are concentrated at the villus tips merely as a result of the physical folding of the epithelium. This leads to $\mathrm{Hh}$ activation in the stroma and differentiation of the epithelium at the villus tips ${ }^{45}$. It is tempting to speculate that, as a result of perturbations in the tissue architecture during tumourigenesis, ligand concentrations are insufficient to activate downstream $\mathrm{Hh}$ signalling in the tumour stroma. Interestingly, in the Apc-shRNA model, the expression of Gli1, Gli2, and Hhip is consistently reduced further in tumours carrying an additional mutation in Kras (Supplementary Fig. 2c), which show higher levels of dysplasia than tumours in Apc-shRNA animals, as well as invasive behaviour, supporting a model in which disrupted tissue architecture interferes with ligand availability and induction of the stromal $\mathrm{Hh}$ response ${ }^{21}$. Moreover, in our hands, AOM/DSS-induced tumours occasionally displayed signs of invasion through the muscularis mucosae (Supplementary Fig. 9 and refs 16,46).

Although the AOM/DSS model recapitulates the Hh expression pattern of human CRC as well as parts of the mutagenic landscape of sporadic human colon cancers ${ }^{16}$, tumour development is accelerated by colitis, which is not a self-evident feature of most human carcinomas. Nevertheless, sporadic colorectal carcinogenesis is characterized by, and is dependent on, a complex inflammatory cell infiltrate and immuno compromised mice are protected from both inflammatoryassociated and sporadic colon tumours ${ }^{47,48}$. On a clinical note, our data indicate that caution is warranted particularly when using $\mathrm{Hh}$ antagonists in patients with inflammatory bowel disease, who are at an increased risk of developing $\mathrm{CRC}^{49}$. However, together with data from pancreatic and bladder cancer, in which $\mathrm{Hh}$ antagonism similarly accelerates carcinogenesis ${ }^{50,51}$, our results point to the possibility that treatment with $\mathrm{Hh}$ antagonists could increase the risk for several solid malignancies, including CRC.

Functionally, the data suggest a dominant, but not exclusive role for BMP modulation as a mediator of stromal-epithelial crosstalk. BMP signalling is active in adenomas, but is downregulated in $\mathrm{CRCs}^{52}$ (Fig. $7 \mathrm{~d}$ ) and thus mirrors the expression pattern of downstream Hh effectors. In sporadic CRC, high GREM1 expression is associated with treatment resistance and inferior survival ${ }^{53}$. Moreover, ectopic epithelial expression of GREM1 causes hereditary mixed polyposis syndrome, which is characterized by the appearance of multiple colonic polyps that frequently progress to carcinomas ${ }^{54}$. This can be modelled in mice, where ectopic Grem 1 expression leads to the reacquisition of stem cell properties in differentiated cells ${ }^{53}$, which are consequently able to initiate intestinal neoplasia. We show that, as a consequence of $\mathrm{Hh}$ activation, attenuated BMP inhibitor expression is conversely associated with diminution of the intestinal stem cell signature (Fig. 5a,b). Thus, it seems plausible that loss of stromal BMP inhibitors due to the activation of stromal Hh signalling (Fig. 5b) may have the potential to restrain colonic tumour initiation and progression (Fig. 7h).

The importance of paracrine $\mathrm{Hh}$ signalling in tumour-stroma cross-talk has been recognized for more than a decade-yet accumulating data now indicate that Hh-driven signals might represent exploitable targets that are not limited to colon cancer therapy, but extend to other cancer types.

\section{Methods}

Mice. C57BL/6 mice (referred to as wt) were obtained from Scanbur, Sweden, and used at $>8$ weeks of age. All mice were on a C57BL/6 background. Gli1 $1^{\text {lacZ } /+}$ and GliCreER ${ }^{T 2}$ mice were a kind gift from Fritz Aberger, whereas R26-LSL-tdTomato (B6.Cg-Gt[ROSA]26Sortm14[CAG-tdTomato]Hze/J) and Colla2CreER (B6.Cg-Tg[Colla2-cre/ERT]7Cpd/J) mice were obtained from the Jackson Laboratory (Bar Harbor, USA). Ptch $1^{f l /}+$ mice have been described previously ${ }^{27}$. For Ihh $\Delta^{\text {Vil }}$ mice, VillinCreER $R^{T 2}$ mice were crossed with Ihh floxfflox mice and Rosa26-ZSGreen mice, to generate VillinCreERT2-ZsGreen-Ih $h^{\text {flox/flox }}$ animals.

Mice receiving DSS were assessed daily in a cumulative score until recovery (weight, movement and body posture, piloerection, skin condition, food consumption, excretions and breathing). Littermates were used as controls and experimental groups were housed in one cage wherever possible. In the case of Cre/ Lox-p models, the control animals lacked either the Cre or floxed alleles or both.

Chemical models of CRC. AOM (Sigma-Aldrich, A5486) at $12.5 \mathrm{mg} \mathrm{kg}^{-1}$ bodyweight was administered intraperitoneally (i.p.) on day 0 and DSS (DB001;

Figure 7 I Diminished Hh signalling in human colonic adenocarcinomas. (a) TCGA analysis (box plots) of Hh ligand expression and (b) expression of Hh downstream targets in 155 human colon carcinomas; $P$-values (Wilcoxon rank-sum test) are indicated in the panels. (c) Correlation between GLI1 expression and expression of HHIP, PTCH1, IHH and SHH; correlation coefficients and P-values are given in the panels. (d) Expression of the BMP targets ID1 and ID2, the BMP inhibitors GREM1 and NOG, and the BMP ligands BMP5 and GDF10. P-values are indicated in the panels. (e) Analysis of Hh pathway expression in patient-derived xenografts, based on bi-species RNA-sequencing data ${ }^{37}$. Hh ligands are expressed exclusively by human tumour cells, whereas transcripts of downstream targets are mostly of murine origin (that is, stromal). Expression of SMO and PTCH1, as well as its homologue PTCH2, is seen in both compartments (middle panel). (f) Illustration of gene expression in human CRC ( $n=8$ ) sorted for tumour/epithelial cells (Epcam ${ }^{+}, \mathrm{CD} 5^{-}$), leukocytes (Epcam ${ }^{-}, \mathrm{CD}_{4}{ }^{+}$) and fibroblasts (double negative). Each column represents expression data from an individual patient for each compartment (based on GEO data set GSE39395). (g) Differential expression of the indicated transcripts in comparison with stromal expression. Red/blue cells indicate significant up-/downregulation in the stroma; fdr < 0.05. (h) Model of Hh signalling in CRC: fibroblasts (grey) express the Hh receptors, PTCH1 and SMO, and respond to tumour cell-derived ligand by activation of canonical Hh signalling. Reduced stromal Hh aticvity is associated with paracrine inhibition of BMP signalling, partly mediated via derepression of GREM1. Tumour cells (orange) are largely incapable of canonical Hh signalling. However, the Hh co-receptor CDON and possibly PTCH1 itself may function as dependence receptors for the ligands, IHH and SHH, and inhibit tumour cell apoptosis. Upon activation of canonical Hh signalling in the stroma, for example, by knockout of PTCH1 (' $X^{\prime}$, lower panel), expression of BMP inhibitors is repressed, whereas expression of BMP ligands such as GDF10 is upregulated, cumulatively leading to enhanced BMP signalling in the tumour cells. Other stromal factors, such as FOXL1 and GPX3, may contribute independently of BMP signalling to the protective effect of active stromal Hh signalling. ${ }^{\star} P<0.05$, ${ }^{\star \star} P<0.01,{ }^{\star \star \star} P<0.001$ and ${ }^{\star \star \star *} P<0.0001$. 
Tdb Consultancy, Sweden) was initiated at a concentration of $2 \%$ on day 5 and was administered for 5 days ${ }^{15}$. DSS cycles were repeated twice with 14 days of normal drinking water in between. All DSS preparations were renewed after 2 days. For the sporadic model, AOM was administered i.p. once a week at $12.5 \mathrm{mg} \mathrm{kg}^{-1}$ body weight for 10 consecutive weeks.

For Ihh $\Delta^{\text {Vil }}$ mice specifically, 10-week-old animals were injected i.p. with $1 \mathrm{mg}$ Tam (T5648; Sigma-Aldrich) for 5 consecutive days. Two weeks after Tam initiation, mice were injected with AOM $\left(10 \mathrm{mg} \mathrm{kg}^{-1}\right)$ and received drinking water supplemented with $1.75 \%$ DSS for 4 days, which was then changed to untreated drinking water. During weeks 5 and 8 , the mice received $1.25 \%$ DSS for 4 and 3 days, respectively. The DSS concentration and treatment duration were adjusted due to augmented weight loss. After 12 weeks, the mice were killed.

Vismodegib and Tam treatment. Vismodegib (V-4050, LC Laboratories) was administered i.p. ${ }^{55,56}$ in dimethyl sulfoxide twice daily at $25 \mathrm{mg} \mathrm{kg}^{-1}$ body weight for 6 days per week.

For intracolonic administration, 4OH-Tam (H6278; Sigma-Aldrich) was dissolved in $99.5 \%$ ethanol, vortexed, further diluted in corn oil (S5007, Sigma-Aldrich) to reach the working concentration and sonicated for $30 \mathrm{~min}$ at $37^{\circ} \mathrm{C}$ in a water bath. The solution was kept at $37^{\circ} \mathrm{C}$ in darkness until it was instilled intraluminally at $1 \mathrm{mg} 25 \mathrm{~g}^{-1}$ bodyweight. Instillation was performed with US guidance under isoflurane anaesthesia (described below). For visualization of the instilled substance with microcomputed tomography $(\mu \mathrm{CT})$ imaging, $0.1 \mathrm{mg} \mathrm{ml}^{-1}$ barium sulphate (Barium Sulphate (\#11381499) 97\%, Alfa Aesar) was added to the Tam/oil suspension. The $\mu \mathrm{CT}$ was performed under isoflurane anaesthesia (see below) using a Quantum FX $\mu \mathrm{CT}$ imaging system (Caliper Life Sciences, Perkin Elmer, Inc.) a few minutes after administration of the Tam/oil/barium mixture.

For systemic labelling experiments in Colla2CreER;R26-LSL-tdTomato and Gli1CreER ${ }^{T 2} ; R 26-L S L$-tdTomato mice, $5 \mathrm{mg}$ of Tam was dissolved in corn oil using sonication at $37^{\circ} \mathrm{C}$ at $20 \mathrm{mg} \mathrm{ml}^{-1}$ and injected once i.p.

Ultrasound. The Vevo 2100 system (Visualsonics, Canada) was used for high-frequency US ${ }^{57}$. Mice were anaesthetized with $1-2 \% \mathrm{v} / \mathrm{v}$ isoflurane (Baxter, KDG9621). Hair over the abdomen was removed using a depilatory cream. The mice were scanned in a supine position on a heating plate $\left(40^{\circ} \mathrm{C}\right)$ with constant cardiovascular monitoring and protective eye ointment. Scans were performed at $40 \mathrm{MHz}$ (probe MS550D; axial resolution $40 \mu \mathrm{m}$ ) using a 3D-motor for $z$ axis resolution ${ }^{57}$. For administration of intraluminal contrast during colonic imaging, a plastic gavage tube was advanced carefully via the anus and small amounts of US gel were injected to inflate the colonic lumen gently under US surveillance. The largest tumour cross-sectional area was determined visually. For 3D scanning, the $z$ resolution was set to $76 \mu \mathrm{m}$ and the whole tumour length was scanned. Tumour outlines were drawn manually for each slice and 3D reconstruction was performed using Visualsonics Vevo software (v1-5.0).

Immunofluorescence. For IF, the colon was removed, faeces were gently washed out with cold PBS and the tissue was immediately frozen on dry ice in Tissue-Tek O.C.T. compound and stored at $-80^{\circ} \mathrm{C}$. Sections were cut using a cryotome at $20-100 \mu \mathrm{m}$ and incubated for $30 \mathrm{~min}$ in PBS with $0.5 \%$ Triton X-100 (SigmaAldrich; T8787) and 0.1\% TOPRO-3 (Invitrogen; T3605). Slides were mounted between two coverslips (Menzel, Nr. $1.5(0.16-0.19 \mathrm{~mm})$ ) and scanned using a Zeiss LSM710 confocal microscope in single-photon mode. For antibody staining, $20-100 \mu \mathrm{m}$ sections were cut in a cryotome and incubated overnight in antibody diluted in PBS plus $0.5 \%$ Triton-X-100, $0.5 \%$ BSA and $0.5 \%$ dimethyl sulfoxide at $4{ }^{\circ} \mathrm{C}$. A species-appropriate secondary antibody (1:250 dilution) was chosen from Alexa Fluor dyes (Invitrogen) using an appropriate wavelength depending on the primary antibody and the presence of endogenous fluorophores. The antibodies and dilutions were as follows: anti-vimentin (Santa Cruz, sc7557, 1:500); anti-desmin (Abcam, ab8592, 1:500); anti- $\alpha$-sma (Abcam, ab5694, 1:500); anti-collagen1 (Abcam, ab34710, 1:200); anti- $\beta$-catenin (Cell Signalling, \#9582, 1:100); anti-Ita6 (CD49f, BD Pharmigen, \# 555734, 1:100).

IHC and X-gal staining. For IHC, the colon was incised longitudinally and fixed in $4 \%$ paraformaldehyde (PFA) in PBS overnight at $4{ }^{\circ} \mathrm{C}$ before routine processing and paraffin embedding. Next, $4 \mu \mathrm{m}$ sections were baked at $60^{\circ} \mathrm{C}$ for $1 \mathrm{~h}$ and rehydrated.

The sections were subjected either to a heat-induced epitope retrieval step or to proteinase-induced epitope retrieval, depending on the primary antibody used. Heat-induced epitope retrieval was performed in citrate buffer ( $\mathrm{pH}$ 6) or DIVA reagent (Biocare Medical, DV2004) using a pressure cooker. Protein-induced epitope retrieval was performed in a $37^{\circ} \mathrm{C}$ water bath using $0.05 \%$ protease (Sigma). Sections were incubated with antibodies against B220 (eBioscience; clone RA3-6B2; 1:200), CD3 (Dako; \#N1580; 1:20), F4/80 (Invitrogen; clone BM8; 1:100), MPO (Dako \#A0398; 1:500), $\beta$-catenin (Cell Signalling; \#9582; 1:100), phospho-smad1/5 (Ser463/465; Cell Signalling; 9516S; 1:50), cleaved caspase-3 (Asp175; Cell Signalling; 9661S; 1:200) or Ki67 (Novocastra; NCL-Ki67p; 1:200) for $1 \mathrm{~h}$ at room temperature (RT). After rinsing, the sections were incubated with a biotinylated secondary antibody: donkey anti-rabbit, rabbit anti-rat antibody
(Dianova) or rabbit anti-goat (Vector, BA-5000), followed by incubation with alkaline phosphatase-labelled streptavidin (Dako; CD3, B220, F4/80, MPO, $\beta$-catenin) for $30 \mathrm{~min}$ at RT or streptavidin peroxidase (Invitrogen; for all others). Alkaline phosphatase was revealed with Fast Red (Dako; AR17911-2) and peroxidase was revealed with DAB (Invitrogen; DAB plus reagent kit; \#002020). Sections were counterstained with haematoxylin or eosin (for X-gal stained tissue) and slides were mounted with water-based mounting medium (Polyscience; \#18606). Negative controls omitted the primary antibodies. Triple-IF was performed with antibodies against desmin (AF3844, R\&D, 1:200) and vimentin (\#5741, Cell Signalling, 1:100), followed by secondary antibodies as described above together with a Cy3-coupled anti-sma antibody (C6198, Sigma Aldrich, 1:200).

$\mathrm{X}$-gal staining was performed as previously described with slight modifications ${ }^{27}$. Briefly, colon tissue was fixed in $2 \%$ PFA/PBS with $0.2 \% \mathrm{v} / \mathrm{v}$ glutaraldehyde for $30 \mathrm{~min}$ at RT. Tissues were washed once for $15 \mathrm{~min}$ in $2 \mathrm{mM}$ $\mathrm{MgCl}$ in PBS plus $0.01 \%$ Nonidet P-40 (BDH Laboratory Supplies; \#56009). Tissues were incubated for $15 \mathrm{~h}$ at $37^{\circ} \mathrm{C}$ in X-gal substrate solution (stock solution: $100 \mathrm{ml}$ of washing buffer, $1 \mathrm{ml}$ of $5 \mathrm{mM}$ potassium ferricyanide, $1 \mathrm{ml}$ of $5 \mathrm{mM}$ potassium ferrocyanide and $2.5 \mathrm{ml}$ of $40 \mathrm{mg} \mathrm{ml}^{-1} \mathrm{X}$-gal (Sigma, B9285) in $\mathrm{N}, \mathrm{N}$-dimethylformamide). Specimens were washed once in PBS, fixed in $4 \%$ PFA/PBS for $4 \mathrm{~h}$ at RT and paraffin embedded.

Microphotographs were taken using a Leica DMLB microscope with a Leica DC300F camera or with a Panoramic MIDI scanner (3DHISTECH), imported with PanoramicViewer software (v.1.15.4). Adobe Photoshop (CS5, v12.1) was used to adjust brightness and contrast. Changes were always applied to the whole image.

The colour deconvolution plug-in for ImageJ was used to quantify DAB and $\mathrm{X}$-gal intensities, and to manually define regions of interest. 'Periphery' was defined as the outermost area with the strongest X-gal staining. Regions were drawn manually and the pixel intensities for DAB (brown) and X-gal (blue) were quantified on an 8-bit scale.

RNA in situ hybridization. The RNAScope 2.0 High Definition Kit (DAB) (\#310035) and the Multiplex Fluorescent Kit (\#320850) were used ${ }^{35}$. The following probes were all from Advanced Cell Diagnostics: Mus musculus leucine-rich repeat containing G protein coupled receptor 5 (Lgr5), \#312171; mRNA M. musculus GLI-Krüppel family member GLI1 (Gli1), mRNA, \#311001; M. musculus Ihh mRNA, \#413096; M. musculus Grem1 mRNA, \#314741; M. musculus Ptch1 mRNA $\# 402811$. Hybridization was performed according to the manufacturer's protocol with slight modifications (pre-treatment 2 was prolonged to $20 \mathrm{~min}$ and the developing time was extended to $15 \mathrm{~min}$ ). Expression was assessed semi-quantitatively as suggested by the manufacturer from 0 (absent), 1 (sporadic positive cells with single spots), 2 (frequent spots with occasional clusters of signal) to 3 (abundant spots and clusters).

Real-time quantitative PCR. A 20-30 mg sample of snap-frozen tissue was disrupted using a VDI 12-homogenizator (VWR International) and mRNA was extracted according to the manufacturer's protocol using an RNeasy midi kit (Qiagen, \#75142). Real-time PCR was performed using the 7500 Fast Real-Time PCR System (Applied Biosystems). Power Green PCR Master Mix (Applied Biosystems; \#4368708) comprised SYBR Green I Dye, AmpliTaq Gold DNA Polymerase, dNTPs, buffer components and ROX as the passive reference dye. The following primers were used: glyceraldehyde-3-phosphate dehydrogenase (Gapdh), forward: 5'-GGTGTGAACGGATTTGGCCGTATTG-3' ${ }^{\prime}$, reverse: 5' -CCGTTGAA TTTGCCGTGAGTGGAGT-3'; Rplp0/ARP, ribosomal protein, large, P0, forward: 5'-TGCACTCTCGCTTTCTGGAGGGTGT-3', reverse: 5'-AATGCAGATGGATC AGCCAGGAAGG-3'; GLI family zinc finger 1 (Glil), forward: 5'-CGTTTAGCA ATGCCAGTGACC- $3^{\prime}$, reverse: $5^{\prime}$-GAGCGAGCTGGGATCTGTGTAG-3'; Ih forward: $5^{\prime}$-GGCTTCGACTGGGTGTATTA- ${ }^{\prime}$, reverse: $5^{\prime}$-CGGTCCAGGAAA ATAAGCAC-3'; Ptch 1 forward: 5'-TTGGGATCAAGCTGAGTGCTG-3', reverse: 5'-CGAGCATAGCCCTGTGGTTCT-3'; Shh forward: $5^{\prime}$-TGGAAGCAGGTTTC GACTGG-3' ${ }^{\prime}$, reverse: $5^{\prime}$-GGAAGGTGAGGAAGTCGCTGT-3' ${ }^{\prime}$. Gli2 forward: 5'-TGAGGAGAGTGTGGAGGCCAGTAGCA-3', reverse: 5'-CCGGGGCTGGA CTGACAAAGC- ${ }^{\prime} ;$; Hip forward: $5^{\prime}$-TAACGGCCCTTTGGTTGGTGGATTT- ${ }^{\prime}$, reverse: 5'-AGCAAAGCCCAGTGACCAAGCAATG-3'; Lgr 5 forward: 5'-CCAAT GGAATAAAGACGACGGCAACA- ${ }^{\prime}$, reverse: $5^{\prime}$-GGGCCTTCAGGTCTTCCT CAAAGTCA-3'; Axin2 forward: 5'-CAGGAGGATGCTGAAGGCTCAAAGC-3', reverse: 5'-CTCAAAAACTGCTCCGCAGGCAAAT-3'; Ihh forward: 5'-GGCTTC GACTGGGTGTATTA- ${ }^{\prime}$, reverse: $5^{\prime}$-CGGTCCAGGAAAATAAGCAC- $3^{\prime}$; Ptch2 forward: $5^{\prime}$-CCCGTGGTAATCCTCGTGGCCTCTAT- $3^{\prime}$, reverse: $5^{\prime}$-TCCA TCAGTCACAGGGGCAAAGGTC-3'; Grem 1 forward: $5^{\prime}$-AGACCTGGAGACCC AGAGTA-3', reverse: $5^{\prime}$-GTGTATGCGGTGCGATTCAT-3'; Nog forward: $5^{\prime}$-AA GGATCTGAACGAGACGCT-3', reverse: 5'-GCGAAGTAGCCATAAAGCCC-3'; Chrd forward: $5^{\prime}$-GATGCTGTTCCCACTGCAC- ${ }^{\prime}$, reverse: GGCCCATCCTCT TGGTCATA- ${ }^{\prime}$; and Bambi forward: $5^{\prime}$-CGCCACTCCAGCTACTTCTT- ${ }^{\prime}$, reverse: 5'-TGAGCAGCATCACAGTAGCA-3'.

Gene expression microarrays. RNA was isolated from $1 \mathrm{~cm}$ of fresh-frozen whole colonic tissue from Colla2CreER;Ptch $1^{f l f l}$ mice $(n=4)$ and controls $(n=4)$ starting $1.5 \mathrm{~cm}$ proximal to the anus. RNA quality was assessed using the Agilent 2200 Tape Station system; RNA integrity numbers $>8$ were considered sufficient. 
Affymetrix Mouse Gene ST 2.0 arrays were used. Robust multi-array average median polish procedures were applied for normalization. For GSEA analyses ${ }^{58}$, the GSEA Java plug-in v2.1.0 was used to probe non-log-transformed normalized expression data with standard settings (with the exception that the permutation type was set to 'gene_set' and gene sets $<15$ genes were permitted). Gene lists were derived either from publications as indicated, from the Molecular Signature Database (www.broadinstitute.org/gsea/msigdb), or from gene ontology searches via the AmiGO2 database v 2.1.4 (www.geneontology.org), curated to include 'manual assertion' and 'experimental evidence', and excluding 'sequence similarity evidence' (see also Supplementary Tables $2-5$ for the gene sets used).

TCGA query. Processed microarray data for colon adenocarcinoma (AgilentG4502A_07_3) were analysed using gene expression data obtained from TCGA $^{2}$ accessed via the UCSC Cancer Browser ${ }^{59}$. In total, the data set obtained included expression data for 155 primary tumours and 19 normal tissue samples. All analyses were performed in R (version 3.1.1). Associations between GLI1 expression and the selected genes were evaluated using Spearman's rank correlation coefficient. The correlations were derived using the rcorr function from the Hmisc package. Differences in expression levels between tumours and normal tissue samples were analysed using the Wilcoxon rank-sum test from the R stats package. Pre-calculated ESTIMATE scores for the TCGA colon adenocarcinoma data were retrieved from the ESTIMATE web repository (http://ibl.mdanderson.org/ estimate/ $)^{60}$

Analysis of GEO data sets. Data for the GEO data sets GSE67186, GSE39395, GSE17840 and GSE29316 were retrieved from the GEO website (http:// www.ncbi.nlm.nih.gov/geo). For RNA-sequencing data (GSE67186), DESeq ${ }^{61}$ was used to normalize the raw count data and to test for differential expression comparing both the control (shRenilla) to each treatment group (shAPC and shAPC/Kras) and the treatment groups to each other. Microarray data from Affymetrix Human Genome U133 Plus 2.0 (GSE39395 and GSE29316) and Affymetrix Mouse Genome 4302.0 arrays (GSE17840) were robust multi-array average normalized using the affy package ${ }^{62}$ and differential expression between treatment groups was analysed using limma ${ }^{63}$. For GSE39395, gene expression in stromal cells was compared with both epithelial cells and leukocytes, whereas in the cases of GSE29316 and GSE17840, Hh-ligand treated cells were compared with untreated or vehicle-treated controls. The expression pattern of a priori selected genes was subsequently analysed and differential expression was called based on an $\mathrm{fdr}<0.05$.

Statistics. All data (except for microarray and sequencing data) were analysed using GraphPad Prism v6.0e and are presented as the mean and s.e.m. unless otherwise specified in the figure legends. Statistical tests are indicated in the figure legends. All $t$-tests were two-sided. The type 1-error rate was set to $5 \%$, except for GSEA, for which results with an fdr $<0.25$ were considered significant as previously suggested ${ }^{58}$.

Study approval. All animal experiments were performed in accordance with local ethical guidelines and with approval from the local ethics committee (the Swedish Board of Agriculture and the Animal Ethics Committee of the Amsterdam Medical Center, The Netherlands).

Data availability. The gene expression data that support the findings of this study have been deposited at GEO (with the accession code GSE67172); expression data referenced in this study are available in GEO (GSE67186 (ref. 21), GSE39395 (ref. 38), GSE17840 (ref. 14) and GSE29316 (ref. 33)). All other data that support the findings of this study are available from the corresponding author (R.T.) upon request.

\section{References}

1. Teglund, S. \& Toftgård, R. Hedgehog beyond medulloblastoma and basal cell carcinoma. Biochim. Biophys. Acta Rev. Cancer 1805, 181-208 (2010).

2. Cancer Genome Atlas Network. Comprehensive molecular characterization of human colon and rectal cancer. Nature 487, 330-337 (2012).

3. Yauch, R. L. et al. A paracrine requirement for hedgehog signalling in cancer. Nature 455, 406-410 (2008).

4. Varnat, F. et al. Human colon cancer epithelial cells harbour active HEDGEHOG-GLI signalling that is essential for tumour growth, recurrence, metastasis and stem cell survival and expansion. EMBO Mol. Med. 1, 338-351 (2009).

5. Büller, N. V. J. A., Rosekrans, S. L., Westerlund, J. \& van den Brink, G. R. Hedgehog signaling and maintenance of homeostasis in the intestinal epithelium. Physiology 27, 148-155 (2012).

6. Scales, S. J. \& de Sauvage, F. J. Mechanisms of Hedgehog pathway activation in cancer and implications for therapy. Trends Pharmacol. Sci. 30, 303-312 (2009).
7. Sekulic, A. et al. Efficacy and safety of vismodegib in advanced basal-cell carcinoma. N. Engl. J. Med. 366, 2171-2179 (2012).

8. Berlin, J. et al. A randomized phase II trial of vismodegib versus placebo with FOLFOX or FOLFIRI and bevacizumab in patients with previously untreated metastatic colorectal cancer. Clin. Cancer Res. 19, 258-267 (2013).

9. Catenacci, D. V. T. et al. Randomized phase Ib/II study of gemcitabine plus placebo or vismodegib, a Hedgehog pathway inhibitor, in patients with metastatic pancreatic cancer. J. Clin. Oncol. 33, 4284-4292 (2015).

10. Herter-Sprie, G. S., Kung, A. L. \& Wong, K. -K. New cast for a new era: preclinical cancer drug development revisited. J. Clin. Invest. 123, 3639-3645 (2013).

11. van Dop, W. A. et al. Loss of Indian Hedgehog activates multiple aspects of a wound healing response in the mouse intestine. Gastroenterology 139, 1665-1676.e10 (2010).

12. Büller, N. V. J. A. et al. Stromal Indian hedgehog signaling is required for intestinal adenoma formation in mice. Gastroenterology 148, 170-180.e6 (2015).

13. Kosinski, C. et al. Indian Hedgehog regulates intestinal stem cell fate through epithelial - mesenchymal interactions during development. Gastroenterology 139, 893-903 (2010).

14. Zacharias, W. J. et al. Hedgehog is an anti-inflammatory epithelial signal for the intestinal lamina propria. Gastroenterology 138, 2368-2377.e4 (2010).

15. Neufert, C., Becker, C. \& Neurath, M. F. An inducible mouse model of colon carcinogenesis for the analysis of sporadic and inflammation-driven tumor progression. Nat. Protoc. 2, 1998-2004 (2007).

16. Fazio, V. et al. The AOM/DSS murine model for the study of colon carcinogenesis: from pathways to diagnosis and therapy studies. J. Carcinog 10, 9 (2011).

17. Hu, Y., Le Leu, R. K. \& Young, G. P. Detection of K-ras mutations in azoxymethane-induced aberrant crypt foci in mice using LNA-mediated real-time PCR clamping and mutant-specific probes. Mutat. Res. Toxicol. Environ. Mutagen 677, 27-32 (2009).

18. Bai, C. B., Auerbach, W., Lee, J. S., Stephen, D. \& Joyner, A. L. Gli2, but not Glil, is required for initial Shh signaling and ectopic activation of the Shh pathway. Development 129, 4753-4761 (2002).

19. Öhlund, D., Elyada, E. \& Tuveson, D. Fibroblast heterogeneity in the cancer wound. J. Exp. Med. 211, 1503-1523 (2014).

20. Zeuner, A., Todaro, M., Stassi, G. \& De Maria, R. Colorectal cancer stem cells: from the crypt to the clinic. Cell Stem Cell 15, 692-705 (2014).

21. Dow, L. E. et al. Apc restoration promotes cellular differentiation and reestablishes crypt homeostasis in colorectal cancer. Cell 161, 1539-1552 (2015).

22. van den Brink, G. R. et al. Sonic hedgehog expression correlates with fundic gland differentiation in the adult gastrointestinal tract. Gut 51, 628-633 (2002).

23. van den Brink, G. R. et al. Indian Hedgehog is an antagonist of Wnt signaling in colonic epithelial cell differentiation. Nat. Genet. 36, 277-282 (2004).

24. Gagné-Sansfaçon, J., Allaire, J. M., Jones, C., Boudreau, F. \& Perreault, N. Loss of sonic Hedgehog leads to alterations in intestinal secretory cell maturation and autophagy. PLoS ONE 9, e98751 (2014).

25. Barbieri, A. et al. Intraluminal gel ultrasound and eco-color doppler: new tools for the study of colorectal cancer in mice. Vivo Athens Greece 27, 443-450 (2013).

26. Zheng, B., Zhang, Z., Black, C. M., de Crombrugghe, B. \& Denton, C. P. Ligand-dependent genetic recombination in fibroblasts: a potentially powerful technique for investigating gene function in fibrosis. Am. J. Pathol. 160, 1609-1617 (2002).

27. Kasper, M. et al. Wounding enhances epidermal tumorigenesis by recruiting hair follicle keratinocytes. Proc. Natl Acad. Sci. USA 108, 4099-4104 (2011).

28. Muñoz, J. et al. The Lgr5 intestinal stem cell signature: robust expression of proposed quiescent ' +4 ' cell markers: transcriptomic and proteomic signature of Lgr5 ${ }^{+}$stem cells. EMBO J. 31, 3079-3091 (2012).

29. Mecklenburg, N. et al. Growth and differentiation factor 10 (Gdf10) is involved in Bergmann glial cell development under Shh regulation. Glia 62, 1713-1723 (2014).

30. Madison, B. B., McKenna, L. B., Dolson, D., Epstein, D. J. \& Kaestner, K. H. FoxF1 and FoxL1 link Hedgehog signaling and the control of epithelial proliferation in the developing stomach and intestine. J. Biol. Chem. 284, 5936-5944 (2009).

31. Perreault, N., Sackett, S. D., Katz, J. P., Furth, E. E. \& Kaestner, K. H. Foxll is a mesenchymal modifier of Min in carcinogenesis of stomach and colon. Genes Dev. 19, 311-315 (2005).

32. Barrett, C. W. et al. Tumor suppressor function of the plasma glutathione peroxidase Gpx3 in colitis-associated carcinoma. Cancer Res. 73, 1245-1255 (2013).

33. Chen, W. et al. Canonical hedgehog signaling augments tumor angiogenesis by induction of VEGF-A in stromal perivascular cells. Proc. Natl Acad. Sci. USA 108, 9589-9594 (2011). 
34. Pelczar, P. et al. Inactivation of Patched1 in mice leads to development of gastrointestinal stromal-like tumors that express Pdgfr $\alpha$ but not kit. Gastroenterology 144, 134-144.e6 (2013).

35. Wang, F. et al. RNAscope: a novel in situ RNA analysis platform for formalin-fixed, paraffin-embedded tissues. J. Mol. Diagn. 14, 22-29 (2012).

36. Mazumdar, T., DeVecchio, J., Agyeman, A., Shi, T. \& Houghton, J. A. The GLI genes as the molecular switch in disrupting Hedgehog signaling in colon cancer. Oncotarget 2, 638-645 (2011).

37. Isella, C. et al. Stromal contribution to the colorectal cancer transcriptome. Nat. Genet. 47, 312-319 (2015).

38. Calon, A. et al. Dependency of colorectal cancer on a TGF- $\beta$-driven program in stromal cells for metastasis initiation. Cancer Cell 22, 571-584 (2012).

39. Walton, K. D. et al. Hedgehog-responsive mesenchymal clusters direct patterning and emergence of intestinal villi. Proc. Natl Acad. Sci. USA 109, 15817-15822 (2012).

40. Mazumdar, T. et al. Hedgehog signaling drives cellular survival in human colon carcinoma cells. Cancer Res. 71, 1092-1102 (2011).

41. Chatel, G. et al. Hedgehog signaling pathway is inactive in colorectal cancer cell lines. Int. J. Cancer 121, 2622-2627 (2007).

42. Delloye-Bourgeois, C. et al. Sonic Hedgehog promotes tumor cell survival by inhibiting CDON pro-apoptotic activity. PLOS Biol. 11, e1001623 (2013).

43. Varnat, F., Zacchetti, G. \& Ruiz i Altaba, A. Hedgehog pathway activity is required for the lethality and intestinal phenotypes of mice with hyperactive Wnt signaling. Mech. Dev. 127, 73-81 (2010).

44. Cohen, M. et al. Ptch1 and Gli regulate Shh signalling dynamics via multiple mechanisms. Nat. Commun. 6, 6709 (2015).

45. Shyer, A. E., Huycke, T. R., Lee, C., Mahadevan, L. \& Tabin, C. J. Bending gradients: how the intestinal stem cell gets its home. Cell 161, 569-580 (2015).

46. Tanaka, T. et al. A novel inflammation-related mouse colon carcinogenesis model induced by azoxymethane and dextran sodium sulfate. Cancer Sci. 94, 965-973 (2003).

47. Quante, M., Varga, J., Wang, T. C. \& Greten, F. R. The gastrointestinal tumor microenvironment. Gastroenterology 145, 63-78 (2013).

48. Whetstone, R. D., Wittel, U. A., Michels, N. M., Gulizia, J. M. \& Gold, B. Colon carcinogenesis in wild type and immune compromised mice after treatment with azoxymethane, and azoxymethane with dextran sodium sulfate. Mol. Carcinog. 55, 1187-1195 (2015).

49. Jess, T., Rungoe, C. \& Peyrin-Biroulet, L. Risk of colorectal cancer in patients with ulcerative colitis: a meta-analysis of population-based cohort studies. Clin. Gastroenterol. Hepatol. 10, 639-645 (2012).

50. Rhim, A. D. et al. Stromal elements act to restrain, rather than support, pancreatic ductal adenocarcinoma. Cancer Cell 25, 735-747 (2014).

51. Shin, K. et al. Hedgehog signaling restrains bladder cancer progression by eliciting stromal production of urothelial differentiation factors. Cancer Cell 26, 521-533 (2014).

52. Kodach, L. L. et al. The bone morphogenetic protein pathway is active in human colon adenomas and inactivated in colorectal cancer. Cancer 112, 300-306 (2008)

53. Davis, H. et al. Aberrant epithelial GREM1 expression initiates colonic tumorigenesis from cells outside the stem cell niche. Nat. Med. 21, 62-70 (2015).

54. Jaeger, E. et al. Hereditary mixed polyposis syndrome is caused by a $40-\mathrm{kb}$ upstream duplication that leads to increased and ectopic expression of the BMP antagonist GREM1. Nat. Genet. 44, 699-703 (2012).

55. Arzumanyan, A. et al. Hedgehog signaling blockade delays hepatocarcinogenesis induced by hepatitis B virus $\mathrm{X}$ protein. Cancer Res. 72 , 5912-5920 (2012).

56. Nagao-Kitamoto, H. et al. GLI2 is a novel therapeutic target for metastasis of osteosarcoma. Int. J. Cancer 136, 1276-1284 (2015).

57. Gerling, M. et al. Real-time assessment of tissue hypoxia in vivo with combined photoacoustics and high-frequency ultrasound. Theranostics 4, 604-613 (2014).

58. Subramanian, A. et al. Gene set enrichment analysis: A knowledge-based approach for interpreting genome-wide expression profiles. Proc. Natl Acad. Sci. USA 102, 15545-15550 (2005).

59. Cerami, E. et al. The cBio Cancer Genomics Portal: an open platform for exploring multidimensional cancer genomics data. Cancer Discov 2, 401-404 (2012).
60. Yoshihara, K. et al. Inferring tumour purity and stromal and immune cell admixture from expression data. Nat. Commun. 4, 2612 (2013).

61. Anders, S. \& Huber, W. Differential expression analysis for sequence coun data. Genome Biol. 11, R106 (2010).

62. Gautier, L., Cope, L., Bolstad, B. M. \& Irizarry, R. A. affy-analysis of Affymetrix GeneChip data at the probe level. Bioinformatics 20, 307-315 (2004).

63. Ritchie, M. E. et al. limma powers differential expression analyses for RNA-sequencing and microarray studies. Nucleic Acids Res. 43, e47-e47 (2015).

\section{Acknowledgements}

This work was supported by the Center for Innovative Medicine (CIMED) at Karolinska Institutet, the Swedish Research Council (K2013-67X-20434-07-4) and the Swedish Cancer Society (\#150665, all to R.T.) as well as the Ruth and Richard Julin Foundation (2015juli44263, to M.G.), Bengt Ihre-Fonden (2012-SLS 254491, to S.A.) and Karolinska Institutet's forskningsfonder (2014fobi42063 to S.A.). M.G. received a postdoctoral stipend from the German Research Foundation (DFG Ge 2386/1-1) and the Swedish Cancer Society (2014/1376). B.E. and L.K. were supported by MD fellowships from the Boehringer Ingelheim Fonds, Germany. L.B. gratefully acknowledges a Marie Curie postdoctoral fellowship (\#297639). We are grateful to Elin Tüksammel and Maryam Saghafian for technical assistance in the animal facility; Tarja Schröder, Contanze Cieluch and Simone Spieckermann for help with IHC; and Agneta Andersson for performing RNA ISH. We thank Maria Kasper for valuable discussions, Anja Füllgrabe for assistance with microarray data processing and Åsa Kolterud for advice on Colla2CreER mice. Parts of this study were performed at the Live Cell Imaging Unit, Department of Biosciences and Nutrition, Karolinska Institutet, supported by grants from the Knut and Alice Wallenberg Foundation, the Swedish Research Council, CIMED, and the Jonasson donation to the School of Technology and Health, Kungliga Tekniska Högskolan, Sweden. We thank the Bioinformatics and Expression Analysis (BEA) core facility, which is supported by the Board of Research at Karolinska Institutet and the Research Committee at the Karolinska Hospital.

\section{Author contributions}

M.G. designed and performed experiments, analysed data and wrote the manuscript N.V.J.A.B., M.C.B.W. and G.R.v.d.B. contributed to experiments with Ihh $\Delta^{\text {Vil }}$ mice and commented on the manuscript. L.K., B.E., Å.B., A.A.K. and L.B. performed experiments and analysed data. R.V.K. analysed histology and commented on the manuscript. S.J. analysed GEO data and commented on the manuscript. O.F. and E.F. analysed TCGA gene expression data and commented on the manuscript. S.A. advised on experimental design and commented on the manuscript. R.T. designed experiments, supervised the study and wrote the manuscript. N.V.J.A.B. and L.K. contributed equally to this work.

\section{Additional information}

Supplementary Information accompanies this paper at http://www.nature.com/ naturecommunications

Competing financial interests: The authors declare no competing financial interests.

Reprints and permission information is available online at http://npg.nature.com/ reprintsandpermissions/

How to cite this article: Gerling, M. et al. Stromal Hedgehog signalling is downregulated in colon cancer and its restoration restrains tumour growth. Nat. Commun. 7:12321 doi: 10.1038/ncomms12321 (2016).

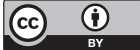

This work is licensed under a Creative Commons Attribution 4.0 International License. The images or other third party material in this article are included in the article's Creative Commons license, unless indicated otherwise in the credit line; if the material is not included under the Creative Commons license, users will need to obtain permission from the license holder to reproduce the material To view a copy of this license, visit http://creativecommons.org/licenses/by/4.0/

(C) The Author(s) 2016 


\section{Erratum: Stromal Hedgehog signalling is}

\section{downregulated in colon cancer and its restoration restrains tumour growth}

Marco Gerling, Nikè V.J.A. Büller, Leonard M. Kirn, Simon Joost, Oliver Frings, Benjamin Englert, Åsa Bergström, Raoul V. Kuiper, Leander Blaas, Mattheus C.B. Wielenga, Sven Almer, Anja A. Kühl, Erik Fredlund, Gijs R. van den Brink \& Rune Toftgård

Nature Communications 7:12321 doi: 10.1038/ncomms12321 (2016); Published 5 Aug 2016; Updated 13 Sep 2016

In Fig. $6 \mathrm{f}$ of this Article, the labelling of the two immunohistochemistry images was inadvertently changed from 'Haematoxylin, ki67' to 'Haematoxylin, Casp-3' during the production process. The correct version of Fig. 6 appears below as Fig. 1. 

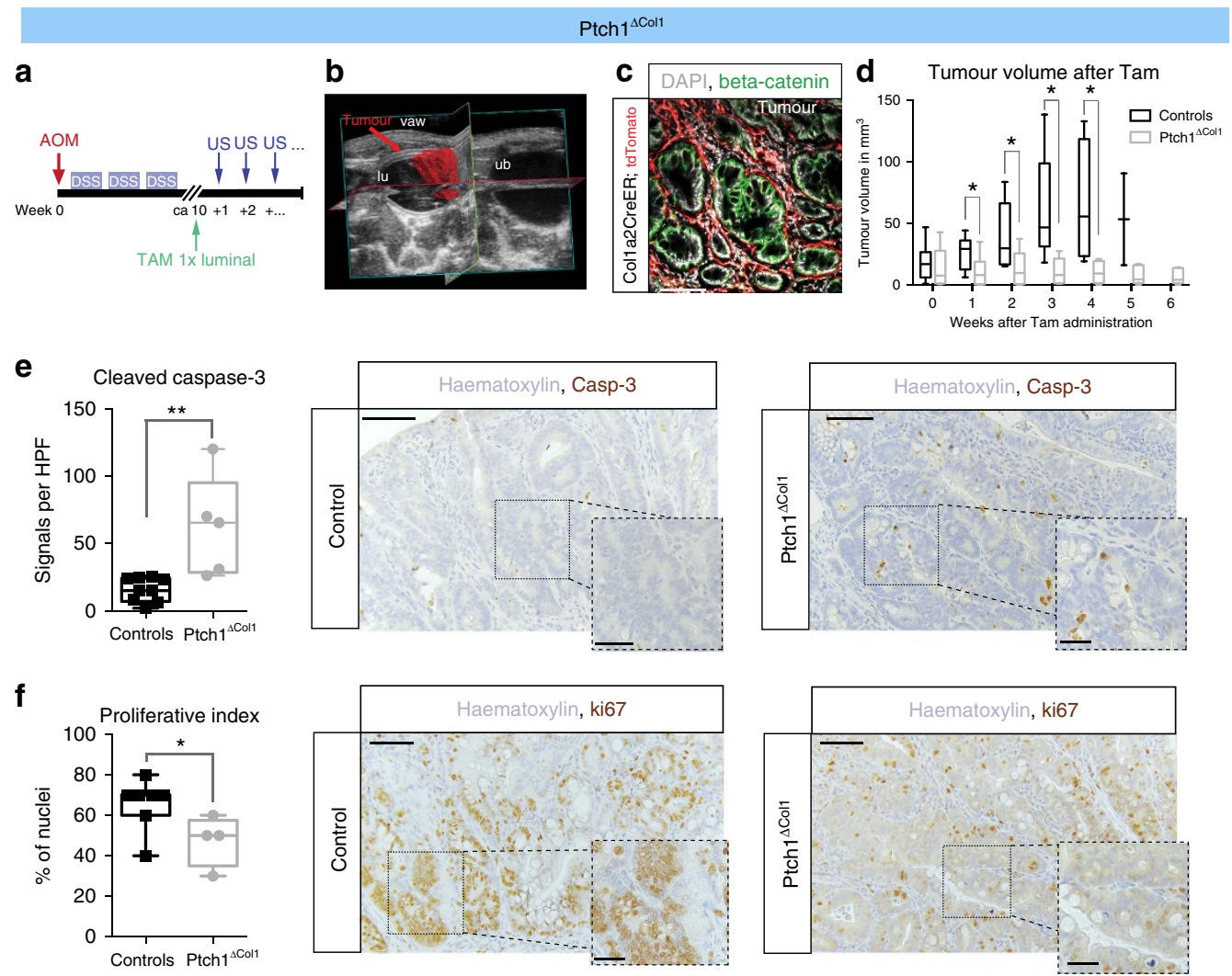

\section{g}
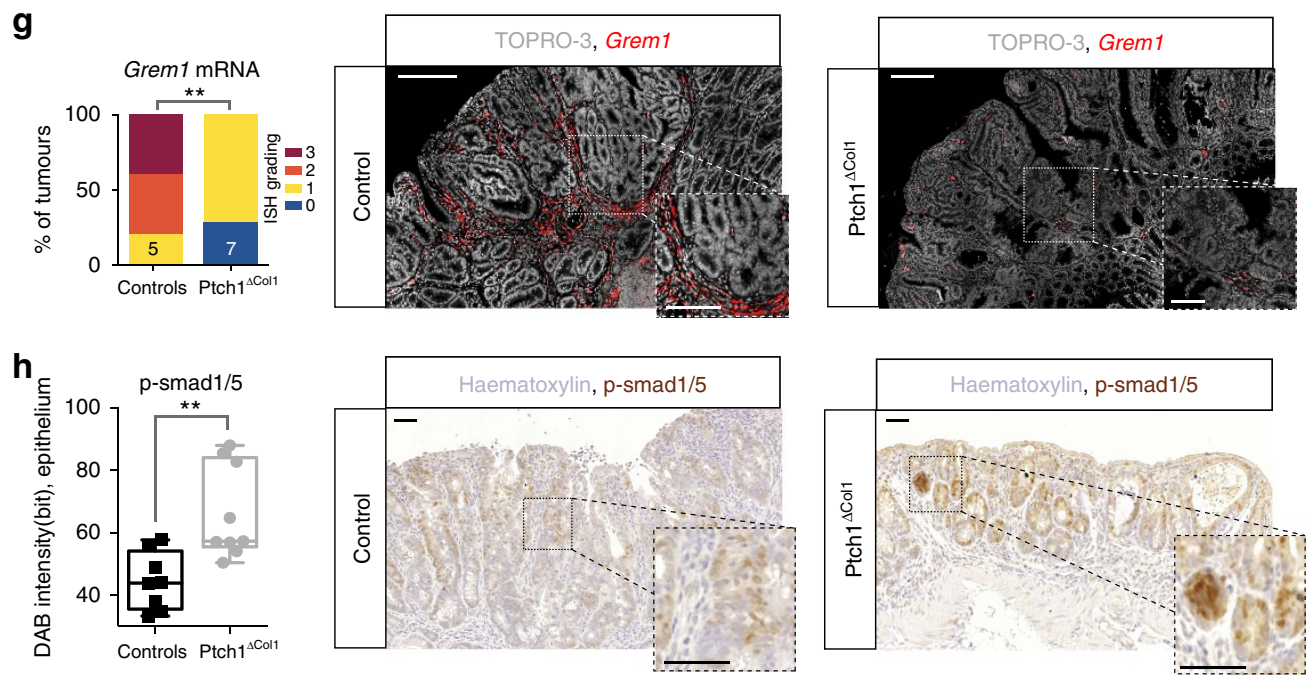

Figure 1

cc (i) This work is licensed under a Creative Commons Attribution 4.0 International License. The images or other third party material in this article are included in the article's Creative Commons license, unless indicated otherwise in the credit line; if the material is not included under the Creative Commons license, users will need to obtain permission from the license holder to reproduce the material. To view a copy of this license, visit http://creativecommons.org/licenses/by/4.0/

(C) The Author(s) 2016 